\title{
The Development of Basic Concepts of Chemical Kinetics in Heterogeneous Catalysis
}

\section{Steps in the development of general chemical kinetics}

How far should one go in search of the sources of science? "The past is a well of ineffable depth" wrote Thomas Mann beginning his novel Joseph and his Brothers. As far as chemical kinetics is concerned, it is far from being true. Certainly, one can trace its remote sources (as far back as Empedocles and Aristotle), but the origin of this science is quite certain; it is the $1850 \mathrm{~s}-1870 \mathrm{~s}$. The basic concepts of chemical kinetics as a science were formulated at that time on the basis of liquid-phase organic reactions.

The pioneers of chemical kinetics Williamson, Wilhelmi, Sainte-Claire Deville, Berthelot, Pean de Sainte Jille and finally the authors of the law of mass action, Guldberg and Waage, had chosen etherification and the reverse reactions of saponification, the reaction of cane sugar transformation, etc. as the subjects of their investigations. As a rule, these reactions were catalytic. Catalysis as a specific chemical phenomenon, consisting of a drastic change of chemical reaction rates in the presence of some substances, had been identified only slightly earlier. In 1836 Berzelius first used the concept of "catalysis" in his paper Some thoughts concerning one agency acting in the formation of organic compounds in living nature but which so far have been unnoticed.

Studies of the "enhancement" of chemical reactions should promote the interpretation of the concept of "reaction rate" and the conduct of special experiments. Probably, one may even say that catalytic investigations have catalyzed the development of chemical kinetics*. Williamson said: "There exist many evidences that chemical processes need time, but this commonly accepted fact is not taken into account in treating various phenomena"**.

\footnotetext{
* Catalysis catalyzed the development of chemical kinetics but did not force its acceptance. Chemical kinetics largely developed on the basis of catalytic reaction data but did not account for the fact that they were catalytic. The development of catalytic kinetics properly started only in the second decade of the 20th century.

** In the description of the basic steps in the development of chemical kinetics we made up our mind to cite many quotations since historical problems are more distinct in the light of direct evidence. On the other hand we believe in the dictum which says that only those who do not cite are those who do not hope to be cited.
} 
Williamson seems to have been the first to use the chemical term "dynamics" with respect to some processes in one of the currently most widespread meanings (non-steady-state processes). The title of Williamson's work, in 1851, was simply Some considerations on chemistry dynamics exemplified by the etherification theory.

The first quantitative relationship for the reaction rate (by the way, also catalytic) was, apparently, first obtained by Wilhelmi in his studies of the effect of acids on cane sugar. The relationship was of the form

$$
-\frac{\mathrm{d} Z}{\mathrm{~d} T}=M Z S
$$

where $Z$ and $S$ are the amounts of sugar and acid catalyst, respectively, $T$ is the reaction time, and $M$ (according to Wilhelmi) the mean amount of sugar which has undergone inversion during an infinitesimal period of time under the effect of unit concentration of the catalyzing acid (the relationship and designations are taken from the book [1, p.14]).

Later, Wilhelmi's study was evaluated by Ostwald who said: "We must consider Wilhelmi as a founder of the chemical reaction rate" [2, p. 28]. But Ostwald admitted that 'Wilhelmi's study had remained absolutely ignored though it was published in a rather widespread Annals of Physics by Poggendorff. .. It remained unknown for the later researchers working on similar problems. .. Only after this field of science had already been so developed that some people began to think about its history did this basic Wilhelmi study come to light" [2, p. 28].

Wilhelmi anticipated some relation of his reaction rate studies with the interpretation of the nature of catalytic action. "I must leave chemists to decide whether the relationships found can be used and, if so, to what extent they are applicable to other chemical processes. In any case, however, I believe among them must be all those processes whose occurrence is ascribed to the catalytic effect" (cited in ref. 3, p. 99).

In 1862-1863 Berthelot and Pean de Sainte-Jille studied the equilibrium states in etherification reactions. In 1862-1867 Guldberg and Waage, on the basis of Berthelot and Pean de Sainte-Jille's experiments and their own data, suggested a primary formulation of the law of mass action.

Reverse reaction equilibrium was represented as a balance of the oppositely acting "affinity" forces

$$
k p q=k^{\prime} p^{\prime} q^{\prime}
$$

where $p, q, p^{\prime}$ and $q^{\prime}$ are the "action masses" of the reactants, and $k$ and $k^{\prime}$ are affinity coefficients, being functions of the "attraction forces" of the reactants. 
In 1879 Guldberg and Waage substituted the above formulation for the basic law of chemical reactions by its modern version in terms of the concept of mobile equilibrium. For the interaction between the initial substances $A$, $\mathrm{B}, \mathrm{C}, \ldots$, taken in the stoichiometric ratio of $\alpha$ to $\beta$ to $\gamma$, i.e. $\alpha \mathrm{A}+\beta \mathrm{B}+\gamma \mathrm{C}$, the reaction rate, $W$, was expressed as

$$
w=K p^{\alpha} q^{\beta} r^{y}
$$

Chemical kinetics had originated in the classical studies by Van't Hoff and Arrhenius in the 1880s. Then the physical sense of reaction orders was interpreted and the concept of activation energy was suggested. The main ideas in Van't Hoff's book [4] are still appropriate.

Van't Hoff suggested the main types of normal chemical transformation. As known, he is the author of the "natural" classification of simple (elementary) reactions according to the number of molecules that are simultaneously present in the reaction. He put forward the principle: "The process of chemical transformation is characterized solely by the number of molecules whose interaction provides this transformation" [4]*.

Van't Hoff himself, however, suggested that the observed chemical transformation follows this principle in rather rare cases. The reason for this discrepancy is the effect of the medium on the reaction rate.

One of the most applicable terms in Van't Hoff's studies was the perturbation actions. In Van't Hoff's opinion, "normal transformations take place very rarely ...". Reaction rate is subjected to various effects to such an extent that the investigation of the transformation process reduces mainly to that of perturbation effects. Van't Hoff did not concentrate on "perturbation effects" as inhomogeneity, non-isothermality, and the occurrence of some secondary reaction. To his mind, the main thing that merits special consideration is the effect of the medium on the reaction rate ("primarily the effect of the media of obviously chemical nature").

That was Van't Hoff's position. For modern kinetics of heterogeneous catalysis his words: "... the effect of the medium on the transformation rate during transformation processes is the most important and the most real" retain their significance.

Van't Hoff also examined the effect of temperature on the course of chemical transformations and drew a fundamental conclusion: "The temperature effect must be gradual and not sudden".

Van't Hoff, and Arrhenius who further developed his ideas, claimed that

\footnotetext{
* Note that at that time the atomic molecular structure of substances had not been proved experimentally. Even two decades later, the great chemist W. Ostwald tried to create "chemistry without molecules",
} 
temperature is not the reason for the reaction but it is responsible for the changes in its rate. Their contributions to chemical kinetics can be compared with that made by Galilei to mechanics: force is not the reason for motion but the reason for acceleration. It is now evident that the conclusions of Van't Hoff and Arrhenius are valid for elementary reactions. As far as complex reactions in open systems are concerned, the dependence of their rates on temperature can also be jumpwise (critical effects).

In his comments on Van't Hoff's book, Semenov wrote "when one is reading this book one feels as if the author was much more interested in the reasons for the abnormal reaction course and the perturbation effect rather than in further extending his knowledge of the normal process, since he treated them as virtually evident ... Van't Hoff's consideration of the abnormal behaviour of reactions is thrice as much" [5, p. 7]. To our mind this splendid principle suggested in Van't Hoff's book should be specially distinguished in modern chemical kinetics. It goes without saying that the sophisticated theory must be supported by accurate experiments*.

The initial period of chemical kinetics (1860-1910) is the key to the understanding of the further progress in this science. It is during this period that formal kinetics was created. The lucidity (and the small number) of the basic conceptions and the integrity of its subject are characteristic of this period of chemical kinetics. Later, that initial integrity was lost, giving way to many forms of "kinetics": gas- and liquid-phase reactions, catalytic, fermentative, electrochemical, topochemical, plasmachemical, and other kinetics. These "kinetics" differ in their experimental techniques and special languages.

Fortunately, the Babylonian tower situation did not repeat itself, since the conceptual ties put forward for the originating chemical kinetics were sufficiently durable. To summarize its two conceptions: (1) the law of mass action as a law for simple reactions and (2) the complexity of chemical reaction mechanisms have remained essential. In order not to exceed the scope of this book, we will consider the Arrhenius temperature dependence, $k(T)=A(T) \exp (-E / R T)$, whose role can hardly be over-estimated. For details, refer to ref. 6 .

The general scientific significance of the law of mass action (l.m.a.) is obvious. Long ago it was applied far beyond the confines of chemical kinetics in so-called "evolution models" [7]. Models based on the l.m.a.-type laws have been applied in biology and ecology [8,9], economics, neurophysiology, genetics and even in military science [7]. Classical "predator-prey" models investigated by Lotka and Volterra in the 1930s go back to the l.m.a. [10-12]. In his description of the dynamics of two interacting populations, Lotka

\footnotetext{
* Not to be trapped, one must remember the wisdom of scientific folklore: the worse the experiment, the more interesting the effect.
} 
proceeded from chemical kinetics models. Biologists, however, do not always remember it*.

The fundamental concept of chemical kinetics is that of reaction mechanism. In the broad sense, the word mechanism ("detailed", "intimate") is the comprehensive interpretation of all experimental data accumulated on the complex reaction process. In this mechanism, one should discriminate individual stages and reaction steps, give characteristics for intermediates, describe transition states of individual steps, provide energy levels of substances, etc. As far as catalytic reactions are concerned, one should characterize surface properties, examine the adsorption character, etc. "I want to know everything" about a complex chemical reaction; this is the way one must understand chemists when they speak about their intention to investigate a detailed mechanism. Whether it is possible to realize such good intentions at a modern theoretical and experimental level will be another question.

For practical chemical kinetics there also exists another (narrow) interpretation of the mechanism as a combination of steps. Each step consists of direct and reverse reactions. If steps are assumed to be simple, they consist of elementary reactions obeying the l.m.a. as their kinetic law, or a surface action law for catalytic reactions.

It is this interpretation of the mechanism that the formal kinetics dealing with kinetic models operates in the form of sets of differential and algebraic equations corresponding to the mechanism.

According to Laidler, an elementary reaction is the reaction taking place to overcome an energy barrier. This barrier is an elementary reaction. An elementary act of the chemical reaction can also be called a chemical transformation taking place between two collisions $[6, \mathrm{p} .19]$.

\footnotetext{
* As a curiosity, let us describe the application of l.m.a. far beyond the confines of common sense. Early in the 20th century, Otto Weininger's book Sex and Character (Principal Investigation) was very popular. In its sixth edition (1914), he wrote: "The law of sexual affinity has many more similarities with one known law of theoretical chemistry but certainly with many deviations (?). It is close to the phenomena associated with the "law of mass action" ... [further, he gives its formula (p. 37)]. At first, the author determines the portion (concentration) of male (M) and female (F) originating from individual $\mathrm{X}$ through $\alpha$ and $\alpha_{1}$ and for individual $\mathrm{Y}$ through $\beta$ and $\beta_{1}$, respectively $\left(\alpha, \beta, \alpha_{1}, \beta_{1}<1\right)$. " $\ldots$ The force of mutual attraction is expressed as: $A=K /(K-\beta) \mathrm{f}(t)$, where $\mathrm{f}(t)$ is some empirical or analytical function during which (?) individuals can be mutually affected, i.e. reaction time as we refer to it and $K$ is the proportionality factor responsible for all known and unknown laws of sexual affinity. In addition, $K$ depends on the degree of population, race and family affinity and also on the health and the absence of defects in both individuals. If, in the above formula, $K=\beta$, then $A=\infty$ (extreme case)". His final conclusion (p.41) is: "So, it is quite evident what I mean: sexual attraction of two individuals being together for a long time or saying it better, locked together, can evolve even where they first had an aversion to one another, which is similar to a chemical process that needs much time until it becomes observable", Although Weininger noted: "It is clear that one cannot attach much significance to the similarities between sexual affinity and dead chemistry", we can hardly believe it.
} 
The Japanese physico-chemist Horiuti in his paper "How can a kinetic equation be found for a reverse reaction?" gives an extremely vivid description of a reaction between the complex and the simple in chemical kinetics. He claims: "We can write an arbitrary number of chemical equations for the same reaction, and each will be accounted for by some equilibrium constant. It can be pleasant to write a chemical equation so that it has the least integer coefficients, but we have no grounds (within the confines of our discussion) to give preference to this chemical equation and its corresponding equilibrium constant.

Previously, when the theorem $\vec{k} / \vec{k}=K$ had been formulated, there were no difficulties of this kind. When we were students, we believed that this theorem is accurately deduced on classical examples of the formation of ethers and hydrogen iodide. Chemical equations with the least integer coefficients were treated as those representing one act in the rearrangement of interatomic bonds, i.e. one elementary reaction, and it was treated as something that went without saying. It is essential that the theorem under consideration is actually valid when one treats a single elementary reaction. We now know, however, that chemical equations are written merely to describe experimental data concerning the material balance without laying any claims to interpret the true mechanism of atomic rearrangements. Having lost the exalted function to represent the mechanism, chemical equations have remained simple expressions for the equivalence of substance totalities to the left and to the right from the equality sign in conformity to the preservation of atoms, like the rate of currency exchange accounts for the preservation of its value. Currency exchange equations, e.g. "four roubles per one dollar" or "one rouble per quarter of a dollar" differ in their coefficients, but this difference is for the sake of convenience in calculations, nothing more. The same holds for chemical equations, and here we are facing the above question, i.e. what is the equilibrium constant in the theorem under consideration?" [13].

Horiuti quotes the American chemist Daniels: "Despite Eyring and Arrhenius, chemical kinetics is all-in-all confusion. But through all the confusion of complications some promising perspective can be seen. Numerous consecutive, competing and reverse reactions by themselves are simple mono- or bimolecular reactions that in principle obey simple laws. Hence we are fighting not so much with primary steps as with the problem of their mutual coordination to interpret the observed facts and to make practical predictions" [13]. Such considerations had been made a very long time ago.

In $1789^{*}$ a professor of chemistry and mineralogy from Dublin (Higgins) for the first time applied the concept of "intermediate substance" in his book Comparative Consideration of Phlogiston and Antiphlogiston Theories when

\footnotetext{
* Thus the year of the Great French Revolution appeared to be portentous for chemical kinetics. Semenov, 150 years later, called chemical kinetics the "chemistry of intermediates".
} 
studying the interaction between iron and chlorous acid. Several years later, Miss Foolgem (in England) came to the conclusion that hydrolytic reactions take place through intermediates. In 1806 Clement and Desorm showed that the oxidation of sulphur in the presence of potassium nitrate (a method of that time to produce $\mathrm{H}_{2} \mathrm{SO}_{4}$ ) is a complex reaction enhanced by the nitric oxides produced in the course of the reactions*.

The years following Van't Hoff's publication [4] are known as a period of rapid progress in the study of multi-step chemical reactions. There appeared Ostwald's and Kistjakovskii's studies, Bach-Engler's peroxide theory, and Luther and Shilov's theory of conjugated reactions. The postulate claiming that "a reaction is not a single-act drama" (Schönbein) had become a common belief.

Of great importance was the principle of independent courses for individual reactions, suggested by Ostwald in 1887. Later, Christiansen compared the problem of elucidating a complex reaction mechanism with that of solving crossword puzzles [14]. There are, however, some other sources of this ideology. As is known, the scientists of the 18th and 19th centuries (including chemists) were respectfully admired for the achievements in mechanics. In 1783, Lavoisier in his paper "Affinity of oxygen origins" wrote: "It is possible that one day the accuracy of the available data will be increased to such an extent that the geometer (in this case it is a mechanician-mathematician) sitting in his study-room will be able to calculate the phenomena accompanying any chemical compound by the same, so to speak, method by which he calculates motions of celestial bodies. Viewpoints expressed in this connection by de Laplace and the experiments we are planning on the basis of his ideas to express the affinity forces numerically already permit us not to consider this hope as some chimera" (quoted in ref. 15). According to Berthollet, "chemical affinity also meets the conditions specified by mechanics for the phenomena depending on the mass-actionlaw" (quoted in ref. 16). It is evident that Guldberg and Waage proceeded from the mechanical interpretation of chemical laws. Primarily it refers to their first studies (1864-1867) in which they gave an "equilibrium" formulation for the law of mass action $\left(k p q=k^{\prime} p^{\prime} q^{\prime}\right)$. Guldberg and Waage wrote: "In chemistry like in mechanics the most natural method will be to determine forces in their equilibrium state" (quoted in ref. 17, p. 341)**.

As a rule, in the literature on the history of chemistry (see, for example,

\footnotetext{
* According to Chugaev, the study of Clement and Desorm was the second piece of evidence in favour of the possible existence of a new class of reactions (catalytic). The first piece was the Dutch chemists' study of the decomposition of ethyl alcohol to water and ethylene in the presence of silica or alumina.

** It should be noted that in their pioneering work in 1864 Guldberg and Waage used an expression whose form is close to the present-day dynamic formulation $\left(w=k p^{\alpha} q^{\beta} r^{3}\right)$ but in the further study "Investigations of chemical affinity" (1867) they decided it would be enough to apply the equilibrium formula $k p q=k^{\prime} p^{\prime} q^{\prime}$.
}

References pp. $80-84$ 
refs. 1 and 16), it is mentioned that, in the initial stages of its development, chemical kinetics operated essentially with mechanical analogies. Particular emphasis is placed upon the analogies applied to deduce the main chemical kinetic law (l.m.a.). However, the fact that the term "complex reaction mechanism" has an obvious "mechanical origin" (i.e. is associated with applied mechanics) remains obscure. In 1879 a vivid interpretation of complex systems as mechanical systems was given by Maxwell: "In an ordinary chime every bell has a rope that is drawn through a hole in the floor into the bell-ringer room. But let us imagine that every rope instead of putting into motion one bell participates in the motion of many parts of the mechanism and that the motion of every bell is determined not only by the motions of its own rope but by the motions of several ropes; then let us assume that all this mechanism is hidden and absolutely unknown for the people standing near the ropes and capable of seeing only the holes in the ceiling above them" $[18, \text { p. 268 }]^{*}$. This image, standing in the light of such known images as Plato's "cave" and the "black box", was just in the spirit of that period. The ways in which scientific terms originate are tortuous. Only a special investigation can lead to the conclusion (not necessarily unambiguous) as to where the term came from, i.e. either from a developed neighbouring science or from a wide common-scientific and even humanitarian-metaphorical context. We now believe it correct to suppose that both the results obtained in theoretical mechanics spurred the construction of the main kinetic law (l.m.a.) and the achievements in applied mechanics gave impetus to dismantle a "complex" chemical reaction into simple details ("by screws"). Finally it led to the development of a constructive conception of a "reaction mechanism". It would be interesting to find out who was the first to apply this term"**

This term was introduced to the normal chemical language in the 20th century due to the efforts of Bodenstein. In Semenov's view, the understanding that, no matter how complicated is a reaction's process the law of the elementary act is sufficiently simple, is exclusively the credit "of Van't Hoff's genius prediction, though he himself did not understand it quite clearly" [5, p. 6]. Though the epithet "genius" with respect to Jacob Henri Van't Hoff is still valid, the situation, however, defies its complete reconstruction. On the one hand, it is likely that Van't Hoff renounced in principle the analysis of complex reactions that do not obey the laws of "normal conversions". Apparently, it is for this reason that in the "Etudes" he did not examine etherification reactions practically [19]. Van't Hoff studied such simple reactions as the decomposition of dibromosuccinic acid and the reaction of

\footnotetext{
* It was in 1879 that Guldberg and Waage's study with a dynamic formulation of l.m.a. was published.

** In the Russian literature, the term "chemical kinetics" was, apparently, first introduced in 1889 by Menshutkin in his book Essays on the Development of Chemical Conceptions.
} 
sodium chloroacetate with sodium hydroxide (mono- and bimolecular reactions, respectively). We have already mentioned that Van't Hoff deliberately did not consider secondary conversions. On the other hand, he unambiguously claims: "As a theoretical foundation I have accepted not the concept of mass action (I had to leave this concept in the course of my experiments)" [4, p. 39].

We do not completely understand with what this quotation is associated since the new value designated by Van't Hoff as concentration had previously been used by Guldberg and Waage ("amounts of these substances with respect to the same volume") [17]. It can be repeated once again that the historic-scientific situation as well as the history itself cannot always be reconstructed.

By the beginning of the 20th century an independent field of physical chemistry, namely chemical kinetics, had been developed. Temkin treats chemical kinetics as a science dealing with chemical reaction rates and specifies the reaction kinetics as "the dependence of the rate of a given reaction on the substance concentration, temperature and some other parameters, e.g. the electrode potential in electrochemical reactions". Semenov interprets chemical kinetics as a science "not only about the rates but also about the mechanism of chemical reactions" [5, p. 9].

In recent years, in studies of the unsteady-state behaviour of chemical systems, the term "dynamics" has been used (see, for example, ref. 20) but its meaning is dubious. First, dynamics is known to be a field of mechanics dealing with the motion of material bodies induced by the applied forces. It is in this sense that the dynamics of the interaction between reacting molecules is treated [21].

Secondly, there is also a wider meaning of the term, i.e. time evolution of the motion* and in this sense the terms "unsteady state" and "dynamic" can be treated as synonyms. The term "dynamic system" refers to a physical system described by a set of differential equations of the type $x=\mathrm{f}(x)$ or even simply to a set of differential equations irrespective of its origin.

The dynamics of chemical reactions is interpreted as a field of the general theory dealing with the evolution of chemical systems on the basis of the dynamic equations for kinetic and mathematical physics [20]. Validity of the use of the term "dynamics of chemical reactions" is primarily due to the fact that it is supported by the extensive use of physical and mathematical methods to investigate dynamic systems. It should be noted that Van't Hoff [4] treated the term "dynamics" in just this sense ("the process of chemical transformation").

\footnotetext{
* It is likely that the meanings of the "motion induced by forces" and "time evolution" have merged.
} 
We have already specified the two significant initial conceptions of chemical kinetics: the law of mass action as a law for simple reactions and the complex character of the mechanism of chemical reactions.

These conceptions have given impetus to the development of two trends that must complement each other: studies of the kinetic regularities of elementary acts and construction of the kinetic theory for complex reactions.

The former trend has led to the development of the collision theories that permitted one to estimate pre-exponential factors of the reaction rate constants and primarily to the elaboration of the absolute rates theory [22].

Based on quantum and statistical mechanics, this theory gave estimates for the parameters of the Arrhenius relationships, i.e. activation energies and pre-exponential factors. The absolute rate theory made use of the suggestion implying the fulfilment of Maxwell-Boltzmann energy distribution of reacting molecules. In recent years the theory and practice of elementary act studies have been developed. The application of a crossed molecular beams method has permitted for the first time the determination of macroscopic parameters (reaction cross-sections and inelastic molecular collisions), to vary energy distribution in individual degrees of freedom for the initial particles, and to record the effect on the probability of chemical transformations and the energy distribution in reaction products.

Today, non-equilibrium reaction theory has been developed. Unlike the absolute rate theory, it does not require the fulfilment of the Maxwell-Boltzmann distribution. Calculations are carried out on large computers, enabling one to obtain abundant information on the dynamics of elementary chemical acts. The present situation is extensively clarified in the proceeddings of two symposia in the U.S.A. [23, 24].

As far as the chemical kinetics of complex reactions is concerned, an important milestone was the chain reaction theory developed by Bodenstein, Semenov and Hinshelwood. It is almost the first theory of complex chemical reactions. A great achievement is that the role of free atoms and radicals has been interpreted on the basis of the analysis of kinetic relationships. Kinetic chemists began to operate with structural "mechanistic" units, i.e. "chains" and "cycles".

As early as at the beginning of the century some difficulties in studying kinetic models corresponding to cyclic schemes were overcome. (In 1902 it was Wegscheider who coped with the problem of the example of the mechanism: (1) $A_{1} \rightleftharpoons A_{2}$; (2) $A_{3} \rightleftharpoons A_{4}$, and (3) $A_{2}+A_{3} \rightleftharpoons A_{1}+A_{4}$.)

For a long time scientists had been discussing the "Wegscheider paradox". In 1931, Onsager, proceeding from the concept of detailed equilibrium in chemical reactions ("chemists apply a very interesting approach"), deduced the known reciprocal relationship, the Onsager equation. Thus these relationships originated from the analysis of cyclic mechanisms in complex reactions. 
The general scientific importance of chain reaction theory is also obvious: it has, for example, stimulated the development of nuclear reaction theory.

A further step in the development of complex reaction theory was the Horiuti-Temkin theory (a theory of steady-state reactions) suggested in the 1950s-1960s. It will be described in detail below. In the age of computers, the kinetics of complex reactions is investigated by modelling. This is the study of processes on the basis of their models. Having omitted the 20 available definitions (among them several philosophical definitions), we will restrict ourselves only to one: "object M is a model of object A with respect to a certain group of characteristics (properties), if $\mathrm{M}$ is constructed (or chosen) to simulate A according to these characteristics". And then: "a mathematical model can be a number, a geometrical image, a function, a set of equations, etc." [25, p. 106].

The mathematical models of chemical kinetics just referred to are, in what follow, the mathematical descriptions that permit us to obtain the dependence of the chemical transformation rate on the reaction parameters (temperature, reactant concentrations, etc.). It is the sole purpose of those models specified as kinetic models [26].

Kinetic models are the basis for the mathematical modelling of chemical reactions. There exists a chain of models that has to be passed through for the calculation of a large catalytic aggregate: kinetic model, catalyst pellet model, catalyst bed model, contact reactor model, and aggregate model. In this hierarchy of models, suggested and thoroughly elaborated by Boreskov and Slinko [27, 28], the kinetic model is the first level. None of the calculations that are of interest for technology can be carried out without kinetic models.

But what must one know before "constructing" any (including kinetic) model? First its basic elements, secondly the main laws and principles of the processes that are to be accounted for by the model, and thirdly the algorithm (the instruction) for the model construction. For kinetic models the basic elements are chemical substances and elementary acts; the main laws are the laws of mass action and surface action; the algorithms for model construction are the methods to derive kinetic equations suggested by Temkin, those to determine kinetic equation constants, etc.

To study kinetic models, one must formulate and solve both direct and reverse kinetic problems.

A direct kinetic problem consists of calculating multi-component reaction mixture compositions and reaction rates on the basis of a given kinetic model (both steady-state and unsteady-state) with the known parameters. Reliable solution for the direct problem is completely dependent on whether these parameters, obtained either on theoretical grounds or from special experiments, have reliable values. Modern computers can solve high-dimensional problems. Both American and Soviet specialists have calculated kinetics for the mechanisms with more than a hundred steps (e.g. the reac- 
tions of hydrocarbon oxidation). The considerable experience accumulated in such calculations was delivered in the proceedings of the 1977 symposium devoted specially to this problem [23]. There remains just one small thing, namely to obtain reliable constants for the models developed.

As known, one of the most extensively investigated complex reactions is the gas-phase oxidation of hydrogen. Kondratiev and Nikitin believe that this reaction must be treated "as a model one ... in some way or another representing an oxidation reaction in general". For hydrogen oxidation in which the number of reactants is not so large $\left(\mathrm{H}_{2}, \mathrm{O}_{2}, \mathrm{H}_{2} \mathrm{O}, \mathrm{H}, \mathrm{O}, \mathrm{OH}, \mathrm{OH}_{2}\right.$, $\mathrm{H}_{2} \mathrm{O}_{2}$, "the third body" being $\mathrm{M}$ ), it is advisible to construct the maximumcompleted mechanism, including all probable steps, if only accounting for the natural stoichiometric limitations (with not more than 3 reacting molecules). Such a maximum mechanism has been suggested by Dimitrov [29] along with the estimates of reaction rate constants. On the basis of the kinetic model corresponding to this mechanism, Dimitrov carried out a numerical investigation of the $\mathrm{H}_{2}+\mathrm{O}_{2}$ system [30].

A reverse kinetic problem consists in identifying the type of kinetic models and their parameters according to experimental (steady-state and unsteady-state) data. So far no universal method to solve reverse problems has been suggested. The solutions are most often obtained by selecting a series of direct problems. Mathematical treatment is preceded by a qualitative analysis of experimental data whose purpose is to reduce drastically the number of hypotheses under consideration [31].

It is now absolutely clear that the computer-aided numerical simulation is not a panacea for the study of complex reactions. An urgent problem is to establish the qualitative effect of the structure of a complex reaction mechanism on its kinetic characteristics. This problem is intimately connected with the classification of mechanisms.

As early as in 1934 Semenov said that "the classification of reactions by their kinetic regularities, even if it is much more complicated than the Mendeleev classification of elements by their properties (the periodic law), seems nevertheless to be possible" [32, p. 538].

"To repeat the route of chemistry in the kinetic aspect", that was the formulation of the problem. To our mind, however, in the 1930s "the rational classification principle", whose appearance was predicted by Semenov, could not be realized. The possibility of solving this problem appeared only in recent times in terms of the concepts of the graph theory and the qualitative theory of differential equations. The analysis of the effect of the mechanism structure on the kinetic regularities of catalytic reactions is one of the connecting subjects in the present study.

Note that these problems, unlike those associated with the elucidation of the kinetic regularities in elementary acts, is not very popular, in particular among kinetic chemists. What are the reasons? It is likely that they are historical and psychological. One must hope that in the near future the situation will change since to understand the way of "assembling" a com- 
plex system of simple "elements" is as important and should be as prestigious as the elucidation of the "element" nature*.

The most important results in chemical kinetics have been obtained with interpenetrating physico-chemical and mathematical conceptions. In what way has this been achieved? Primarily through the cooperation of experts in various fields of science; Guldberg, a theoretical chemist and applied mathematician who never dealt with experiment, and chemist Waage, a scrupulous experimenter who formulated the law of mass action. "Guldberg and Waage have shown the way to apply mathematical laws in chemical sciences"; this is the way that their contemporary, English chemist Mouir, estimated their study of "the chemical affinity" immediately after its publication (quoted in ref. 17, p. 347).

Almost concurrently the same formulation was suggested by Harkurt (a mathematician) and Esson (a chemist).

At the present time, the fruitful cooperation of a biophysicist and a mathematician (Zhabotinskii and Korzukhin) has led to the decoding of kinetic oscillations. Certainly, a happy combination of various fruits in one personality is also possible, an example of such a person being Van't Hoff. ("This double inclination to mathematics on the one hand and to chemistry on the other manifested itself in all my scientific interests".) FranckKamenetskii, Horiuti, Semenov and Temkin are also examples of such a combination**.

We believe it to be indisputable that just this combination fits the spirit of chemical kinetics.

\section{The development of the kinetics of heterogeneous catalysis}

We believe the development of heterogeneous catalytic reaction kinetics is determined by the interaction of two mutually supplementing programs.

(1) A program to construct kinetic models of heterogeneous catalytic reactions that would be similar to the generally accepted models of chemical kinetics. This general kinetic model has been implemented in the model of the ideal adsorbed layer.

(2) A program to construct kinetic models accounting for the specificity

\footnotetext{
* Chemical kinetics is not an exciusion. According to Engelhardt, in modern biology the reduction principle, i.e. an elementaristic approach ("separate and cognize") still dominates. But at present the emphasis should be placed on a system and an integrative approach must be developed. "It is high time to claim that the integrative approach is not only the route but also the aim"; that is Engelhardt's idea [33].

** In 1934, Semenov wrote: "A new field can be developed by the joint efforts of physicists and chemists" [5, p.5], but still he himself was afraid of "being accused of superfluous formalism by chemists". There is every reason to believe that his fears were well grounded.
} 
of heterogeneous catalysis. This specific program has been implemented in various theories of catalyst inhomogeneity (Temkin, Roginskii, Zeldovich), of the effect of the reaction medium on the catalyst (Boreskov), etc. Here we can consider a model of the real adsorbed layer.

\subsection{IDEAL ADSORBED LAYER MODEL}

The foundation for heterogeneous catalytic kinetics was laid down in the classical studies of Langmuir [34,35]. and Temkin [36]. It is from these studies that the ideal adsorbed layer model has originated on the basis of the similarity with the concepts of homogeneous kinetics. This model implies (1) the equivalence of all sites of the catalyst surface and the independence of chemisorption energy of surface coverage with various adsorbents, (2) the unchangeability of the catalyst and the independence of its properties of the reaction mixture composition and its influence on the catalyst, and (3) the equilibrium distribution of energy.

The surface action law deduced by Temkin on the basis of the absolute rate theory [36] is of the form

$$
W=A_{\mathrm{e}}^{-\varepsilon_{0} / K T} z_{1}^{\beta_{1}} z_{2}^{\beta_{2}} \ldots p_{1}^{b_{1}} p_{2}^{b_{2}} \ldots z_{0}^{m_{\mathrm{t}}-\sum_{j} \beta_{j}}
$$

Here $A$ is the pre-exponential factor, $\varepsilon_{0}$ the excess energy of the complex activated compared with the energy of the initial particles, $K$ the Boltzmann constant, $z_{j}$ the fraction of the surface occupied by the $j$-type adsorbed particles, $z_{0}$ the free surface fraction, $p_{i}$ the partial pressures of gaseous substances, and $m_{i}$ the number of elementary sites occupied by the activated complex. An expression to calculate the pre-exponential factor $A$ has been given elsewhere [36]*.

At first it was believed that the main factor responsible for the kinetic regularities is the displacement or the "competition" of reaction mixture components for the catalyst surface sites. An additional assumption was made concerning the high rate of the adsorption and desorption steps compared with the chemical transformations proper.

Further investigations showed significant disadvantages of the above assumptions. Nevertheless, Hinshelwood, Schwab, Hougen, Watson and others derived equations which adequately described a particular kinetic experiment within a certain range of parameters.

A typical form of the kinetic equation corresponding to the above assumptions is

\footnotetext{
* Substantiation and deduction of the surface-action law (according to Temkin) can also be found in the monograph by Snagovskii and Ostrovskii [37]. Estimates of pre-exponential factors are given in Krylov's study [38]. One can also apply the methods to calculate $A$ suggested by Golodets and Roiter [39]. To calculate the rate of heterogeneous catalytic reaction one also needs to estimate the number of active sites given by Maatman $[40,41]$ (see also refs. 38 and 42).
} 


$$
W=\frac{k \prod_{i=1}^{N} c_{i}}{1+\sum_{i=1}^{N} K_{\mathrm{p} i} c_{i}}
$$

Here $W$ is the reaction rate, $k$ the rate coefficient, $c_{i}$ the concentration of the $i$ th reactant of the gas phase, and $K_{p i}$ the equilibrium constant for the adsorption step of the $i$ th gas component on the catalytic surface.

Later, it became clear that the concentrations of surface substances must be treated not as an equilibrium but as a pseudo-steady state with respect to the substance concentrations in the gas phase. According to Bodenstein, the pseudo-steady state of intermediates is the equality of their formation and consumption rates (a strict analysis of the conception of "pseudo-steady states", in particular for catalytic reactions, will be given later). The assumption of the pseudo-steady state which serves as a basis for the derivation of kinetic equations for most commercial catalysts led to kinetic equations that are practically identical to eqn. (4). The difference is that the denominator is no longer an equilibrium constant for adsorption-desorption steps but, in general, they are the sums of the products of rate constants for elementary reactions in the detailed mechanism. The parameters of these equations for some typical mechanisms will be analysed below.

The most general description for the kinetics of complex reactions in terms of the ideal adsorbed layer model was given in the Horiuti-Temkin steady-state reaction theory [43-47] (see Chap. 1).

\subsection{REAL ADSORBED LAYER MODELS}

Heterogeneous catalytic reactions are the combinations of interrelated physical and chemical elementary acts in "reaction mixture - catalyst" systems. Here one should discriminate between microscopic and macroscopic kinetics.

In this connection kinetic models can also be separated into microscopic and macroscopic models. The relations between these models are established through statistical physics equations. Microscopic models utilize the concepts of reaction cross-sections (differential and complete) and microscopic rate constants. An accurate calculation of reaction cross-sections is a problem of statistical mechanics. Macroscopic models utilize macroscopic rates.

To determine the latter, a function for the energy distributions between molecules must be known. A detailed consideration of the relations between macroscopic and microscopic parameters can be found in refs. 48 and 49 .

It has been known for a fairly long time that the reaction rate must depend on the law of energy distribution between reacting molecules. Apparently it was Marcelin who first realized this in 1915 [48, p. 149]. Experiments with molecular beams in the 1960 s and 1970 s revealed that, in gas-phase systems, a wide variety of reactions take place that cannot be interpreted without 
taking into account a significant distribution in the degrees of freedom [50, 51]. Here an important problem is to elucidate the degree of non-equilibrium of the function for the energy distributions during chemical reactions*.

The degree of non-equilibrium is determined by the ratio of microscopic rates of reaction to relaxation. Here relaxation is treated as restoration of the Boltzmann distribution due to various physical processes of energy exchange.

As far as the reactions in solids (in particular heterogeneous catalytic reactions) are concerned, there exists an additional relaxation channel, namely the solid body. The rate of energy exchange with solids is high. In principle we can agree with Nikitin's theory [50] that the concept of preservation of the equilibrium distribution here is sufficiently good.

Zhdanov and Zamaraev [53] examined the possibility of non-equilibrium effects for several typical surface reactions. They established some concrete reasons responsible for the fact that, in molecular and bimolecular reactions taking place on solid surfaces, these effects are usually small.

Catalytic reaction steps. The principal steps of complex heterogeneous catalytic reactions are: (1) interactions between the components of the reaction mixture and the catalytic surface, i.e. adsorption and desorption of atoms and molecules and impact interactions between the molecules of gaseous substances and those adsorbed on the surface, (2) processes on the catalyst surface, i.e. interactions between various surface substances in the adsorbed layer, migration of atoms and molecules, changes in the surface state during the reaction, etc., (3) mass transfer processes into the catalyst bulk and dissolution of substances in the near-surface layer, (4) phase and structural catalyst transformations, and (5) energy processes, i.e. energy exchange between reactants and catalyst.

\footnotetext{
* Estimates of the non-equilibrium correction for the rate constants have been reported in several studies. Let us give it in the form recently suggested by Zhdanov [52] for the bimolecular reaction

$\mathrm{A}+\mathrm{A} \rightarrow \mathrm{B}+\mathrm{C}$

A pseudo-steady state distribution function was determined from Boltzmann's equation as

$f(v)=f_{0}(v)+f_{1}(v)$
}

where $f_{0}(v)$ is Maxwell's function and $f_{1}(v)$ is a small correction. The reaction rate constant is $k=k_{0}(1-\eta)$, where $k_{0}$ is the reaction rate constant calculated using the Maxwell distribution function and $\eta$ is characteristic of non-equilibrium.

$k_{0}=\sigma_{\mathrm{r}}^{0} v \exp \left(-E_{\mathrm{a}} / k T\right)$

where $\sigma_{\mathrm{r}}^{0}$ is the reaction cross-section using the Maxwell distribution function, $v=(4 k T / \pi m)^{1 / 2}$, $m$ is the molecular weight, and $E_{\mathrm{a}}$ the activation energy.

$\eta=\left(81 \times 3^{1 / 2} \sigma_{\mathrm{r}}^{0} v_{0} / 8 \pi \sigma_{\mathrm{e}} v\right)\left(k T / E_{\mathrm{a}}\right)^{2} \exp \left(-E_{\mathrm{a}} / 3 k T\right)$

where $\sigma_{\mathrm{e}}$ is the elastic scattering section and $v_{0}$ the minimum velocity of the relative motions of two molecules enabling the reaction. 
Models accounting for catalyst inhomogeneity. The above ideal adsorbed layer model is only a first but necessary approximation for the construction of a theory to describe the kinetic regularities of heterogeneous catalytic reactions. To begin with, it appeared that the assumption of equivalence of all catalyst surface sites is considerably limited. In the 1930s and 1940s, the inhomogeneity of even one-component catalysts was proved by the combination of calorimetric, isotope and other methods. Still earlier, Taylor had emphasized the significance of this fact for catalysis [54]. It has been established that, in most cases, heats of adsorption and desorption activation energies are significantly dependent on the degree of surface coverage with adsorbed substances. It can be due first to the initial difference in the arrangement of atoms on the surface (various crystallographic indices, edges, angles, dislocations, defects, admixtures) and, secondly to the interaction of adsorbed particles or the effect of pre-adsorbed particles on the electron properties of the catalysts. These factors are respectively specified as biographical and induced inhomogeneities of the catalyst. These terms are due to Roginskii.

Inhomogeneity exerts a significant effect on the equilibrium and kinetic relationships of adsorption-desorption processes. For biographically inhomogeneous surfaces, the ideal adsorbed layer model is applied only to an infinitesimal portion of surface sites having the same properties. Then a certain inhomogeneity distribution is suggested and integration over all the types of surface sites is carried out. The Soviet school (Temkin, Roginskii and Zeldovich) devoted a large number of investigations to the dependence of the forms of equilibrium and kinetic adsorption regularities on the chemisorption character. Two problems were under investigation: (1) direct, i.e. the analysis of the effect of a given inhomogeneity type on the shape of the equilibrium adsorption isotherms and kinetic equations and (2) the reverse problem, i.e. the determination of inhomogeneity distribution functions from experimental data. Thus, Temkin, by admitting the non-linear character of inhomogeneity, showed that the Langmuir isotherm is substituted by the logarithmic isotherm equation (Temkin's isotherm) [55]. Having assumed that changes in the adsorption activation energies for various surface sites account for the same portions of adsorption heat, Temkin derived an equation [56] for the adsorption rate that appeared to be identical to the Zeldovich-Roginskii empirical equation [57]. Zeldovich had shown [58] that the exponential character of inhomogeneity leads to Freundlich's isotherm.

Proceeding from an assumption of the linear energy inhomogeneity, kinetic equations were derived for some important industrial processes, primarily the Temkin-Pyzhev equation for ammonia synthesis [59]. A theory for the adsorption and catalytic processes on inhomogeneous surfaces was suggested by Roginskii [60]. The present-day state of the problem and, which is most important, the experience accumulated in the use of kinetic models accounting for the biographical inhomogeneity had been reported by Snagovskii and Ostrovskii [37] and by Kiperman [42, 61]. Deviations from the 
surface-action law in the above models are primarily observed by the fact that kinetic equations are powers with fractional exponents that remain unchanged over a wide range of the parameters. A survey of the kinetic models accounting for the biographical inhomogeneity can be found in refs. 37 and 61 .

As far as the models accounting for the induced inhomogeneity are concerned, they utilize various physical concepts on the interaction of adsorbed particles. Thus Boudart, in his electrostatistical model [62], described a layer of chemisorbed particles as a charged capacitor. The potential difference between the capacitor plates depends on the degree of surface coverage. The dipole-dipole interaction model dating back to Langmuir [63] and de Boer [64] accounts for the interaction of discrete charges of adsorbed particles. Finally, Temkin, in his surface electron gas model [65], suggested that gas adsorption on the catalyst surface changes the electron density in the layer directly adjoining the surface. Therefore, with increasing surface coverage, the activation energy also changes. Accounting for changes in the reaction heat and activation energy by increasing surface coverage is the specific feature of the induced inhomogeneity models. These are applied to treat critical effects discovered experimentally in the kinetic relationships for oxidation reactions.

\subsection{MODELS ACCOUNTING FOR PHASE AND STRUCTURAL TRANSFORMATIONS OF CATALYSTS}

The development of a theory accounting for catalyst inhomogeneity is determined by the availability of a great number of experimental data (calorimetric, isotopic, etc.) that give evidence to this inhomogeneity. At present, numerous qualitatively new experimental data have been accumulated that are to be theoretically substantiated [66, 67].

\subsubsection{Phenomenological model}

First of all, it is clear that the reaction medium can affect a catalyst thus altering its properties. One must pay attention primarily to the studies performed by Boreskov and his school, who suggested a concept of the effect of the reaction mixture on the catalyst. The concept implies that this effect can also be outside the scope of complex reaction steps on the surface. A large number of experimental facts testifying to the changes in the catalyst properties as a result of varying the reaction mixture composition can be found in refs. 68 and 69.

Changes in the catalytic activity of unit accessible surface or the specific catalytic activity (SCA) has attracted the attention of researchers. In the 1950s Boreskov formulated a rule for the approximate constancy of the SCA. According to this rule, the SCA for several metal and oxide catalysts remains approximately constant with significant variations of the surface and crystallite sizes under preparative conditions. Boreskov ascribed this con- 
stancy to the fact that, under the effect of reaction medium, the catalyst is brought to the same steady state irrespective of the initial state of the surface determined by the preparation and pretreatment conditions. In further studies, however, significant deviations from the rule of SCA approximate constancy were found. Boudart et al. in their studies of the dependence between the SCA of metals and their dispersity [69], discovered a change in the SCA by two orders of magnitude and distinguished a class of structural-sensitive reactions. The analysis made by Boreskov [70], however, led him to the conclusion that; despite the initial difference in the SCA of various metal planes, the effect of the reaction medium is observed, as a rule, in smoothing the catalytic properties of these planes. Surface structure is reconstructed towards attaining the energy state that will correspond to the minimum free energy.

The problem of the effect of the reaction medium is important for the understanding of the action of commercial catalysts. Thus, for most reactions of catalytic oxidation on oxide catalysts which depend on the reaction mixture composition, a significant change is observed in the oxygen content and the charge of catalyst cations. This leads to variations in their activity and selectivity $[71,72]$. Slow relaxation in the rate of ethylene oxidation on silver due to the variations of the oxygen content in the near-surface layer is observed [73]. Changes in the composition are often accompanied by reconstruction of the surface structure [74] and finally by the change in the catalyst phase composition [75]. A survey of the recent achievements in studying morphological change of metal catalysts is given in ref. 76 .

Boreskov suggested a phenomenological equation to characterize the reaction medium effect qualitatively [70]

$$
W=\mathrm{f}(c, \theta) R(c)
$$

where $f(c, \theta)$ is the kinetic characteristics at constant catalyst composition, $R(c)$ the parameter responsible for the alteration of properties under the effect of the reaction mixture and $c, \theta$ the concentrations of gaseous and surface substances, respectively. It should be noted that reaction kinetics with variable catalyst activity is described by extensively used models having two constituents: (1) independent of the catalyst state and (2) dependent on it.

Thus, when constructing a kinetic model for the synthesis of vinyl chloride on the "HgCl-coal" catalyst, the following postulates were used: (a) the type of the kinetic equations is independent of the concentration of the active salts; (b) changes in the catalyst activity in any case (mercuric chloride deactivation, removal, etc.) can be treated simply as changes in the active salt concentration [77].

The kinetic model that fits the above requirements is of the form

$$
\frac{\partial x}{\partial \tau}=k\left(c_{\mathrm{HgCl}_{2}}\right) \mathrm{F}(x, T)
$$




$$
\frac{\partial k}{\partial t}\left(c_{\mathrm{HgCl}_{2}}\right)=\psi\left[x, T, k\left(c_{\mathrm{HgCl}_{2}}\right)\right]
$$

where $x$ is the degree of conversion of the acetylene, $T$ the temperature, $c_{\mathrm{HgCl}_{2}}$ the active salt concentration, $\tau$ the contact time, and $t$ the time.

The equation suggested by Boreskov accounts for the presence in the catalytic system of two time scales, namely a "fast" one due to the surface chemical transformations and a "slow" one due to the effect of the reaction mixture on the catalyst. (It shoud be noted that, in general, one can hardly discriminate between the constituents in the way it has been done in this phenomenological equation.)

The construction of phenomenological models accounting for phase and structural catalyst transformations was urgent. However, the situation has now changed significantly in the sense that radically new experimental data have appeared. These were obtained over the last 20 years by using various physical methods, e.g. thermodesorption, IR and Auger electron spectroscopy (AES), low-energy electron diffraction (LEED), low-energy electron loss spectroscopy (LEELS), ion scattering spectroscopy (ISS), secondary ion mass spectroscopy (SIMS), X-ray and ultraviolet photoelectron spectroscopy (XPS and UPS) or electron spectroscopy for chemical analyses (ESCA), etc. Thus we can now give an answer to questions concerning the elementary surface composition. Until recently even the formulation of this problem was impossible.

Methods of studying surfaces based predominantly on electron and ion spectroscopy are becoming increasingly perfect.

It is the extensive application of new physical methods that determines the modern step of "surface science". According to Kopetskii [78], this science is now in a position close to that of condensed state physics early in the 20th century and semiconductor physics during the period 1945-1950.

The development of "surface science" is quantitatively represented as a diagram in (Fig. 1) Bonzel's study [79]. At present a gap exists between the range of parameters (primarily that of high vacuum) for "surface science" (both "ideal" and "extended") which is under development now, and for technical catalysis operating with normal and high pressures. One usually speaks about a "pressure gap", i.e. the gap existing between these two regions. The gap is, however, being reduced, a situation which is promoted by the distinct recent tendency to combine various methods in one apparatus whose combination will permit us to examine catalytic reactions in a wide parametric range $\left(10^{-8}\right.$ to $10^{5}$ Torr $)$.

One of the most interesting observations of "surface science" is the ordered arrangement of adsorbed atoms and molecules (see, for example, refs. 80-84). Numerous LEED experiments showed that the diffraction patterns for partially covered surfaces exhibit some additional spots (additional compared with the absence of adsorbate). These spots correspond to new 


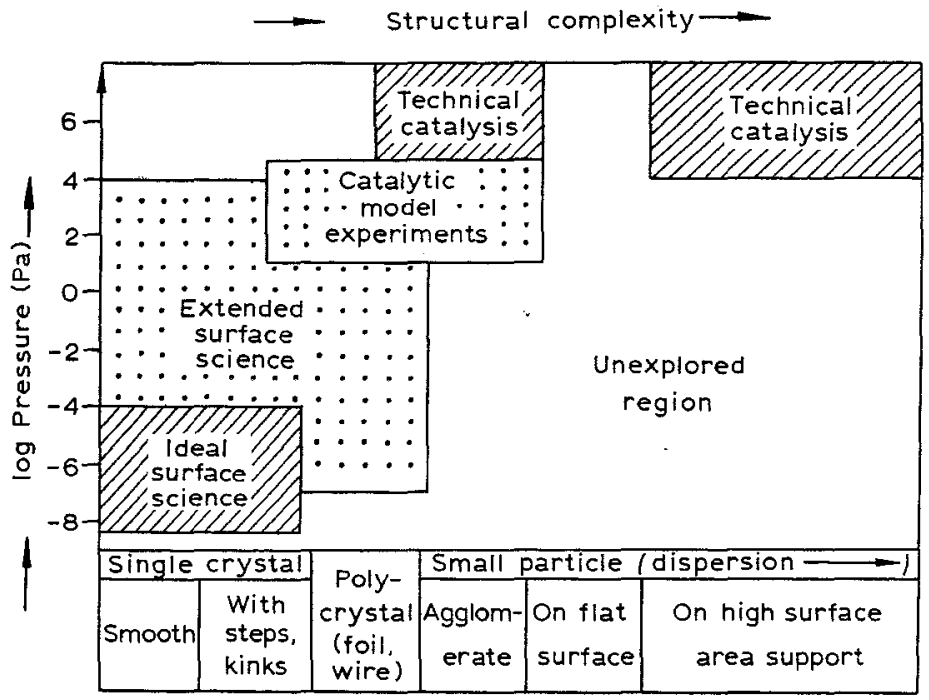

Fig. 1. Development of "surface science" [79].

structures. An atlas of the structures obtained experimentally is given in ref. 85 .

\subsubsection{Lattice gas model}

Recently, in order to understand processes on the catalyst surface, in particular structural formations, it has become a frequent practice to apply theories accounting for the interaction of adsorbed atoms. An important microscopic model of such a type is the lattice gas model*. Its specific peculiarity is that this model accounts for the interaction of the nearer surface molecules (lateral interactions). It is this model that was applied in refs, 86 and 87 . They should be specially emphasized as having exerted a great influence on the interpretation of thermodesorption experiments. The lattice gas model is used, e.g. in a series of investigations by Tovbin and Fedyanin $[88,89]$ devoted to the kinetics of chemisorption and reactions on catalyst surfaces. In terms of this model, one can interpret the complicated reaction rate dependences of surface coverage observed experimentally

\footnotetext{
* The interaction between adsorbed particles was also taken into account in terms of some models of induced inhomogeneity (see the above representation), e.g. in de Boer's dipole-dipole interaction model [64], but compared with the lattice gas model, they must be treated as semi-empirical. A semi-empirical model for the collective interaction of adsorbed particles with catalyst surface was also suggested by Snagovskii and Ostrovskii [37].
} 
[90-92]. The lattice gas model is used to elucidate the "diffusion order-disorder" transition on catalyst surfaces [92-102]. Finally, as has been mentioned already, this model is important in decoding thermodesorption spectra.

The most general formulas to describe the effect of lateral interaction between adsorbed molecules on the rate constants of various processes on solid surfaces were derived by Zhdanov [103, 104]. In particular, the rate constant of the Langmuir-Hinshelwood bimolecular reaction $\mathrm{A}+\mathrm{B} \rightarrow \mathrm{C}$ is determined by the equations [103]

$$
\begin{aligned}
\frac{\mathrm{d} N_{\mathrm{A}}}{\mathrm{d} t} & =\frac{\mathrm{d} N_{\mathrm{B}}}{\mathrm{d} t}=-K\left(\theta_{\mathrm{A}}, \theta_{\mathrm{B}}\right) \hat{N}_{\mathrm{O}} \\
K & =\frac{k T}{2 \pi \hbar} \frac{F_{\mathrm{A}^{*} \mathrm{~B}^{\star}}}{F_{\mathrm{A}} F_{\mathrm{B}}} \frac{z}{2} \sum_{i} P_{\mathrm{AB}, i} \exp \left(-\frac{E_{\mathrm{A}}+\Delta \varepsilon_{i}}{k T}\right)
\end{aligned}
$$

Here $N_{\mathrm{A}}$ and $N_{\mathrm{B}}$ are the concentrations of molecules $\mathrm{A}$ and $\mathrm{B}$ on the surface, $N_{0}$ is the number of elementary cells per unit surface, $F_{\mathrm{A}}, F_{\mathrm{B}}$ and $F_{\mathrm{A}^{*} \mathrm{~B}^{*}}$ are the non-configurational statistical sums of molecules $\mathrm{A}$ and $\mathrm{B}$ and of activated complex $\mathrm{A}^{*} \mathrm{~B}^{*}, z$ is the number of neighbouring cells (for a square cell $z=4$ ), $P_{\mathrm{AB}, i}$ is the probability of two neighbouring cells being occupied by the $A B$ pair and for this pair to have the environment marked by the index $i, E_{\mathrm{A}}$ is the energy difference between the pair $\mathrm{AB}$ and the activated complex $\mathrm{A}^{*} \mathrm{~B}^{*}$; provided that the cells that are nearer to both $\mathrm{AB}$ and $\mathrm{A}^{*} \mathrm{~B}^{*}$ are not occupied, and $\Delta \varepsilon_{i}$ is the energy difference between the interactions of both $\mathrm{A}^{*} \mathrm{~B}^{*}$ and $\mathrm{AB}$ with the environment. It is assumed that molecules $\mathrm{A}$ and $\mathrm{B}$ occupy one elementary cell and the activated complex occupies two of them.

An accurate calculation of the probability in eqns. (5) and (6) is impossible. The most convenient method for an approximate calculation of the probabilities of various configurations is the quasi-chemical approximation that is the simplest version of the cluster approximation. The latter suggests (1) separation of a cluster consisting of several cells, (2) substitution of the interaction between cluster molecules and environmental molecules by some average interaction and (3) application of Gibbs' distribution to calculate the number and distribution of cluster particles. In the quasi-chemical approximation the cluster is minimal, i.e. it consists of two cells. According to this approximation, the probabilities $P_{\mathrm{AB}, i}$ are expressed through $P_{\mathrm{AB}}$, $P_{\mathrm{AA}}$, etc., where $P_{\mathrm{AA}}$ is the probability of two neighbouring cells being occupied by the pair AA, etc. These probabilities fit the set of equations [103]

$$
\begin{aligned}
& P_{\mathrm{AA}}+P_{\mathrm{AB}}+P_{\mathrm{AO}}+P_{\mathrm{BB}}+P_{\mathrm{BO}}+P_{\mathrm{OO}}=1 \\
& 2 P_{\mathrm{AA}}+\mathrm{P}_{\mathrm{AB}}+P_{\mathrm{AO}}=2 \theta_{\mathrm{A}} \\
& 2 P_{\mathrm{BB}}+P_{\mathrm{AB}}+P_{\mathrm{BO}}=2 \theta_{\mathrm{B}} \\
& \frac{P_{\mathrm{AA}} P_{\mathrm{OO}}}{P_{\mathrm{AO}}^{2}}=0.25 \exp \left(-\varepsilon_{\mathrm{AA}} / k T\right)
\end{aligned}
$$




$$
\begin{aligned}
& \frac{P_{\mathrm{BB}} P_{\mathrm{OO}}}{P_{\mathrm{BO}}^{2}}=0.25 \exp \left(-\varepsilon_{\mathrm{BB}} / k T\right) \\
& \frac{P_{\mathrm{AA}} P_{\mathrm{BB}}}{P_{\mathrm{AB}}^{2}}=0.25 \exp \left(\frac{-\varepsilon_{\mathrm{AA}}+\varepsilon_{\mathrm{BB}}-2 \varepsilon_{\mathrm{AB}}}{k T}\right)
\end{aligned}
$$

Equations (7) naturally generalize the well-known equations of the quasichemical approximation for adsorbed molecules of the same type.

According to the quasi-chemical approximation, different pairs are treated as being independent, hence the probability that, for example, $n$ particles of $\mathrm{A}$ and $m$ particles of $\mathrm{B}$ are localized near the particle $\mathrm{A}$, is

$$
P_{\mathrm{A}, n m}=\frac{z !}{n ! m !(z-n-m) !} \frac{P_{\mathrm{AA}}^{n} \bar{P}_{\mathrm{AB}}^{m} \bar{P}_{\mathrm{AO}}^{z-n-m}}{\left(\mathrm{P}_{\mathrm{AA}}+\overline{\mathrm{P}}_{\mathrm{AB}}+\overline{\mathrm{P}}_{\mathrm{AO}}\right)^{z}}
$$

where $\bar{P}_{\mathrm{AB}}=0.5 P_{\mathrm{AB}}$ and $\bar{P}_{\mathrm{AO}}=0.5 P_{\mathrm{AO}}$ [103]. From this one can readily obtain the sum in eqn. (6)

$$
\begin{aligned}
& \sum_{i} P_{\mathrm{AB}, i} \exp \left(-\frac{\Delta \varepsilon_{i}}{k T}\right) \\
& =P_{\mathrm{AB}}\left(\frac{P_{\mathrm{AA}} \exp \left[\left(\varepsilon_{\mathrm{AA}}-\varepsilon_{\mathrm{A}^{*} \mathrm{~A}}\right) / k T\right]+\bar{P}_{\mathrm{AB}} \exp \left[\left(\varepsilon_{\mathrm{AB}}-\varepsilon_{\mathrm{A}^{*} \mathrm{~B}}\right) / k T\right]+\bar{P}_{\mathrm{AO}}}{\mathrm{P}_{\mathrm{AA}}+\bar{P}_{\mathrm{AB}}+\bar{P}_{\mathrm{AO}}}\right)^{z-1} \\
& \quad \times\left(\frac{P_{\mathrm{BB}} \exp \left[\left(\varepsilon_{\mathrm{BB}}-\varepsilon_{\mathrm{B}^{*} \mathrm{~B}}\right) / k T\right]+\bar{P}_{\mathrm{AB}} \exp \left[\left(\varepsilon_{\mathrm{AB}}-\varepsilon_{\mathrm{B}^{*} \mathrm{~A}}\right) / k T\right]+\bar{P}_{\mathrm{BO}}}{P_{\mathrm{BB}}+\bar{P}_{\mathrm{AB}}+\bar{P}_{\mathrm{BO}}}\right)^{z}
\end{aligned}
$$

where $\varepsilon_{\mathrm{AA}}, \ldots$, etc. are lateral interaction energies.

Equations similar to eqns. (5), (6) and (8) were obtained by Zhdanov [104] to describe the monomolecular adsorption and associative desorption and Eley-Rideal's bimolecular reaction. He examined the dependence of the rate constants of these processes on the surface coverages and discussed various approximations applied previously to describe the effect of lateral interaction of adsorbed molecules on the desorption rate constant. He also considered the effect of the lateral interaction on the pre-exponential factor of the rate constants for various processes, and in terms of the "precursor state" model, the effect of ordering the adsorbed molecules on the sticking coefficient and the rate constant of monomolecular desorption.

As usual, the rate of dissociative adsorption (e.g. of $\mathrm{O}_{2}$ on various metals $[92,95,99,100])$ rapidly decreases with increasing surface coverage. As a rule, this is attributed to the fact that dissociative adsorption requires two unoccupied cells, i.e. the sticking coefficient must be $S(\theta)=S\left(\theta_{0}\right) P_{o s}(\theta)$. If a solid surface adsorbs only molecules $\mathrm{A}$, in the quasi-chemical approximation we will have the set of equations

$$
\begin{aligned}
& P_{\mathrm{AA}}+P_{\mathrm{AO}}+P_{\mathrm{BO}}=1 \\
& 2 P_{\mathrm{AA}}+P_{\mathrm{AO}}=2 \theta
\end{aligned}
$$




$$
\frac{P_{\mathrm{AA}} P_{\mathrm{OO}}}{P_{\mathrm{AO}}^{2}}=\frac{1}{4} \exp \left(-\frac{E_{\mathrm{AA}}}{k T}\right)
$$

From eqns. (9)-(11) we obtain

$$
P_{\mathrm{OO}}(\theta)=\frac{1-\theta-(1 / 2)\left\{1-\left[1-4\left(1-\exp \left(-E_{\mathrm{AA}} / k T\right)\right) \theta(1-\theta)\right]^{1 / 2}\right\}}{1-\exp \left(-E_{\mathrm{AA}} / k T\right)}
$$

Equation (12) was presented in ref. 104. The exact solution has been obtained only for a square cell and $\theta=0.5$ [101].

The probability for repulsion is

$$
P_{\mathrm{OO}}\left(\frac{1}{2}, \frac{E_{\mathrm{AA}}}{k T}\right)=\frac{(1-\mathrm{F}(x))}{4},
$$

where

$$
\begin{aligned}
& \mathrm{F}(x)=\left\{\begin{array}{l}
(1+x)^{1 / 2}\left(\frac{1-x}{\pi} K(x)+1 / 2\right), x=\sinh ^{-2}\left(\frac{E_{\mathrm{AA}}}{k T}\right), T<T_{\mathrm{c}} \\
\left(\frac{1+x}{x}\right)^{1 / 2}\left(\frac{x-1}{\pi} K(x)+1 / 2\right), x=\sinh ^{2}\left(\frac{E_{\mathrm{AA}}}{k T}\right), T>T_{\mathrm{c}}
\end{array}\right. \\
& \sinh \frac{E_{\mathrm{AA}}}{2 k T_{\mathrm{c}}}=1 \\
& K(x)=\int_{0}^{\pi / 2}\left(1-x^{2} \sin ^{2} \phi\right)^{1 / 2} \mathrm{~d} \phi
\end{aligned}
$$

where $T_{c}$ is the critical temperature.

The quasi-chemical approximation gives only qualitative results and appears to be particularly inaccurate at temperatures below the "orderdisorder" phase transition points of $T=0.567 E_{\mathrm{AA}}$ at $\theta=1 / 2$.

Thus one can draw the conclusion that the lattice gas model taking into account the interaction of neighbouring molecules is only a first step in studying the effect of adsorbed particle interaction on the rate of heterogeneous catalytic reactions.

The real interaction of chemisorbed molecules, however, can be rather complicated [82,93]. In particular, the parameters of the interaction between neighbouring molecules can change with varying surface coverages due to the collective interaction of adsorbed molecules with solid surfaces. Therefore the lattice gas model that utilizes a small number of parameters cannot describe, even qualitatively, the entirety of surface processes. Nevertheless, this model can be applied to interpret some individual regularities, 
e.g. reaction rate variations with increasing surface coverage and the effect of a phase transition on the reaction rate.

Various approximation of this model (e.g. those of molecular splitting, pseudo-chemical, chaotic and molecular field approximations) were reported and analyzed by Tovbin and Fedyanin $[88,89]$. In particular, the pseudochemical approximation for chemisorption kinetics is of the form

$$
\begin{aligned}
\frac{\mathrm{d} \theta_{\mathrm{A}}}{\mathrm{d} t} & =K_{\mathrm{A}}(1-\theta)\left(\frac{1+x t}{1+x_{1} t}\right)^{z}-K_{\mathrm{D}} \phi(1+x t)^{2} \\
t & =1-\frac{2(1-\theta)}{1+\delta} \\
\delta & =\left\{(1-2 \theta)^{2}+4 \theta(1-\theta) \exp (\beta \varepsilon)\right\}^{1 / 2} \\
K_{\mathrm{A}} & =w_{\mathrm{A}} \exp \left[-\beta \varepsilon_{\mathrm{A}}(\theta)\right]=\frac{J^{*} P}{J_{\mathrm{D}} \hbar \sigma} \exp \left\{-\beta\left[\varepsilon_{\mathrm{A}}+A \theta(1-\gamma)\right]\right\} \\
K_{\mathrm{D}} & =w_{\mathrm{D}} \exp \left[-\beta \varepsilon_{\mathrm{D}}(\theta)\right]=\frac{J^{*}}{J_{\mathrm{a}} \hbar \sigma \beta} \exp \left[-\beta\left(\varepsilon_{\mathrm{D}}-A \theta \gamma\right)\right]
\end{aligned}
$$

Here $J^{*}, J_{0}$ and $J_{\mathrm{a}}$ are the statistical sums of activated complex and gasphase molecules and of adsorbed atom (adatom), respectively, $\varepsilon_{\mathrm{A}}$ and $\varepsilon_{\mathrm{D}}$ the adsorption and desorption activation energies, $\sigma$ the area of adatom localization, $\hbar$ Planck's constant, $\varepsilon_{1}$ and $\varepsilon$ the parameters of the activated complexadatom and adatom-adatom interactions $(\varepsilon<0$ for repulsion and $\varepsilon>0$ for attraction), $A$ the contribution to the complete drop of adsorption heat $\Delta Q$ from the electron subsystem (for a two-dimensional free-electron gas model), $x=\exp \beta\left(\varepsilon_{1}-\varepsilon\right)-1, x_{1}=x\left(\varepsilon_{1}=0\right), \beta=1 / k T$ ( $k$ is the Boltzmann constant), and $\gamma$ the factor relating changes in the activation energy with varying the adsorption heat (for more detail refer to refs. 88 and 89).

The above equations account for the local interaction of adsorbed particles and their collective interaction with the catalyst surface.

A promising study of the lattice gas model is the computer statistical tests (by the Monte Carlo method). Such calculations have been carried out since the mid-1960s (see, for example, refs. 66 and 105). For calculations of gas adsorption on metals, see refs. 106-110. However, no systematic application of the Monte Carlo method to heterogeneous reactions has been carried out: it is to be done in the future.

\subsubsection{Topochemical models}

Phase transformations in heterogeneous catalysis have been described recently by topochemical kinetic models [111-115]. These models were taken from solid chemistry, where they had been developed for "gas-solid" reactions. The products of such reactions are solids. When gas is in contact with the initial solid, the reaction rate is negligible. But as nucleates of the phase 
of a new product are formed, a solid phase interface is formed. The surface of this interface increases with time primarily due to the growth of nucleates (a slow formation of new nuclei also takes place). The reaction rate is at first low but then it increases. Plotted kinetic dependences are S-shaped. The nuclei then coalesce and the reaction rate attains its maximum value.

A simple topochemical model for the growth of $\mathrm{NiO}$ islands on the $\mathrm{Ni}$ surface during the reaction of oxygen with a $\mathrm{Ni}(111)$ crystal is clearly described by Holloway and Hudson [112]. They considered three cases in which the rate-determining step is, respectively (a) oxygen sorption from the gas phase (surface diffusion is fast), (b) surface diffusion of oxygen, and (c) oxygen insertion over the island boundary.

To obtain an expression for the growth kinetics, the following assumptions were made: (1) the surface contains physically adsorbed oxygen. Its sticking coefficient is close to unity, the average lifetime is $\tau$, the surface diffusion coefficient is $D_{\mathrm{s}}$, (2) the probability of oxygen dissociation is low everywhere except at the island boundary, (3) NiO islands are circular, (4) the main lifetime of adsorbed substance and the diffusion coefficient for the surface covered by chemisorbed oxygen and $\mathrm{NiO}$ are the same, and (5) the formation time of the islands is small compared with the total time of their growth.

The area of a unit circular island originating at a moment $\eta$ and then growing, will, at time $t$, be

$$
s_{\eta}=\pi u^{2}(t-\eta)^{2}
$$

where $u=\mathrm{d} r / \mathrm{d} t$ is the linear radial rate of island growth and $u$ is a constant.

The area of all the islands, provided they do not overlap and no new nucleates are generated on the sites where the NiO phase was formed, is

$$
\theta_{\eta}=\int_{0}^{t} \pi u^{2}(t-\eta)^{2} i \mathrm{~d} \eta
$$

Here $i$ is the formation rate of new phase nucleates on a unit surface. It can be shown [111] that the overall oxygen surface coverage, without taking into account the above assumptions, is

$$
\theta=1-\exp \left(-\theta_{\eta}\right)=1-\exp \left(-\int_{0}^{t} \pi u^{2}(t-\eta)^{2} i d \eta\right)
$$

To integrate eqn. (15), one needs expressions for $u$ and $i$. Let us first consider the island growth rate, $u$.

We have suggested that $\tau$ and $D_{\mathrm{s}}$ are similar for the surface covered with both chemisorbed oxygen and $\mathrm{NiO}$. If the reaction rate were determined by the impact of gas-phase oxygen molecules with the surface, it would be constant. But in experiments [112] no constant reaction rate was observed, therefore this limiting case has not been considered: 
Let us assume that the growth of a new oxide phase is controlled by surface diffusion. The number of impacts of oxygen molecules on a unit surface is $I,\left(\mathrm{~cm}^{-2} \mathrm{~s}^{-4}\right)$; the number of impacts on one surface site is $I A$, where $A$ is the area of one surface site. If one assumes that migration over the surface is random, the number of molecules reacting at the island boundary due to the surface diffusion is $4 I A^{1 / 2}\left(D_{\mathrm{s}} \tau\right)^{1 / 2}$.

The rate of arrival of oxygen molecules from the gas phase to the site near the island boundary is determined as $I A^{1 / 2} h$ where $h$ is the height of the island. The rate of island radius variations in the case where the growth is controlled by surface diffusion is

$$
u_{\mathrm{r}}=\frac{8 A I}{n}\left(D_{\mathrm{s}} \tau\right)^{1 / 2}+\frac{2 A h I}{n}
$$

where $n$ is the number of oxide layers in the island.

Finally, let us consider the case in which the rate-determining step is oxygen insertion over the island boundary. If the rate constant of this insertion is $k$, the insertion rate is $I k \tau A$. If one also assumes that molecules come directly to the sites near the boundary, then

$$
u=\frac{4 A^{3 / 2}}{n} I k \tau+\frac{2 A h I}{n} .
$$

The coefficients $D_{\mathrm{s}}, \tau$ and $k$ are determined as

$$
\begin{aligned}
D_{\mathrm{s}} & =z \alpha^{2} v_{1} \exp \left(-\frac{E_{\mathrm{d}}}{R T}\right) \\
\tau & =\frac{1}{\nu_{2}} \exp \left(\frac{E_{\mathrm{a}}}{R T}\right) \\
k & =v_{1} \exp \left(-\frac{E_{\mathrm{in}}}{K T}\right)
\end{aligned}
$$

where $v_{1}$ and $v_{2}$ are the fluctuation frequencies parallel and normal to the surface, $\alpha$ is the mean length of one jump, and $z$ is the reversal to the number of nearer neighbouring sites where the adsorption takes place. Hence, the expressions corresponding to the two cases under consideration can be written as

$$
u_{i}=\frac{K_{i}^{1 / 2}}{\pi^{1 / 2}} P
$$

where

$$
\begin{aligned}
K_{i}^{1 / 2} & =2(A / n) I\left[2 B_{i} \exp \left(E_{i} / R T\right)+h\right] \\
B_{1} & =2\left(z \alpha^{2} v_{1} / v_{2}\right)^{1 / 2} \\
E_{1} & =1 / 2\left(E_{\mathrm{a}}-E_{\mathrm{d}}\right)
\end{aligned}
$$




$$
\begin{aligned}
& B_{2}=A^{1 / 2} \nu_{1} / v_{2} \\
& E_{2}=E_{\mathrm{a}}-E_{\text {in }}
\end{aligned}
$$

and $E_{\mathrm{a}}, E_{\mathrm{d}}, E_{\text {in }}$ are the activation energies of oxygen physical adsorption, its diffusion, and insertion over the island boundary, respectively.

The number of gas molecule impacts per unit surface is determined for an ideal gas as

$$
I=\left(2 m k T_{\mathrm{g}}\right)^{-1 / 2}
$$

where $T_{\mathrm{g}}$ is the gas temperature.

We have suggested previously that the formation time of nucleates is much lower than that obtained experimentally. At time $t=0$ there exist $N_{0}$ sites and the probability of nucleate formation per unit time on a single site is $v$. The formation rate of nucleates at time $\eta$ is

$$
i=\frac{\mathrm{d} N}{\mathrm{~d} t}=N_{\mathrm{o}} v \exp (-v \eta)
$$

After substituting the expression for $u$ and $i$ in the equation for the determination of oxygen surface coverage and integrating by parts, we obtain (assuming that $v t$ is very high)

$$
1-\theta=\exp \left(-K_{i} N_{\circ} p_{\mathrm{O}_{2}}^{2} t^{2}\right)
$$

where $p_{\mathrm{O}_{2}}$ is the partial pressure of oxygen.

The concentration of surface defects is estimated to be $10^{11}$ defects $\mathrm{cm}^{-2}$.

The topochemical model [112] suggests that an island can have $n$ oxide layers. Apparently, this model can be applied in the case of the chemisorbed two-dimensional phase growth, as had been done by Boreskov et al. [116].

The effect of metal structure and phase formation on the kinetics of catalytic oxidation reactions was treated in detail by Savchenko et al. (see, for example, refs. 83, 84, 117 and 118). In metal surface layers both reconstruction of the metal proper (faceting) and processes associated with the formation of surface oxides can take place. In this case the first to form can be chemisorption structures (without breaking the metal-metal bond) and then the formation of two-dimensional surface oxides is observed. Finally, three-dimensional subsurface oxides are produced. An important role is played by the temperature of disordering the adsorbed layer.

When analyzing the oxidation of hydrogen on nickel, Savchenko et al. [117] came to the conclusion that, if the reaction temperature is above that of the disordering of the adsorbed layer (in this case the oxygen layer), it will be quite correct to apply models based on the surface-action law. Otherwise one must take into consideration the "island" character of the interaction.

It should be said that at present the available literature concerning the kinetic models which account for the topochemical character of catalyst surface processes is limited, but reference can be made to refs. 119 and 120 . In ref. 119, a kinetic model for the oxidation of hydrogen on platinum is 
suggested which takes into consideration the interaction on both the sites that are practically free of adsorbates and the boundaries of spots formed by chemisorbed oxygen. In ref. 120, the authors describe a kinetic model for hydrogen oxidation on the $\mathrm{Ni}(110)$ plane corresponding to the detailed mechanism

$$
\begin{aligned}
& \mathrm{O}_{2}+2 \mathrm{Z} \rightarrow 2 \mathrm{ZO} \\
& \mathrm{H}_{2}+2 \mathrm{Z} \rightleftarrows 2 \mathrm{ZH} \\
& \mathrm{ZO}+\mathrm{ZH} \rightarrow \mathrm{ZOH}+\mathrm{Z} \\
& \mathrm{ZOH}+\mathrm{ZH} \rightarrow 2 \mathrm{Z}+\mathrm{H}_{2} \mathrm{O}
\end{aligned}
$$

and account for the topochemical character of $\mathrm{OH}$ groups' interaction with hydrogen atoms adsorbed on the surface.

Let us emphasize the following: as a rule, the literature lacks a strict discrimination between the structures that are formed at the microscopic and macroscopic levels. It is important to understand whether the terms "structure", "ordering" and "islands" refer to microstructures or macrostructures. Thus, the lattice gas model is aimed at accounting for the formation of microstructures, peculiarities in thermodesorption spectra, etc.

The topochemical model, however, describes the origination and growth of macrostructures. In principle one could construct kinetic models accounting for the kinetics of cluster (or nucleate) formation as a model for the system or reverse consecutive reactions [114, 121].

$$
\begin{aligned}
& \mathrm{A}+\mathrm{A} \rightleftarrows \mathrm{A}_{2} \\
& \mathrm{~A}_{2}+\mathrm{A} \rightleftarrows \mathrm{A}_{3} \\
& \mathrm{~A}_{i-1}+\mathrm{A} \rightleftarrows \mathrm{A}_{i}
\end{aligned}
$$

A general form of the respective microscopic equations is given in ref. 122. But one can draw the conclusion that at present no models are known that would account for the origination and growth of clusters and would be constructed on the basis of a correct microscopic description. Their elaboration is a problem of the future.

\subsection{MODELS ACCOUNTING FOR DIFFUSIONAL MASS TRANSFER}

Diffusional mass transfer processes can be essential in complex catalytic reactions. The role of diffusion inside a porous catalyst pellet, its effect on the observed reaction rate, activation energy, etc. (see, for example, ref. 123 and the fundamental work of Aris [124]) have been studied in detail, but so far several studies report only on models accounting for the diffusion of material on the catalyst surface and the surface-to-bulk material exchange. We will describe only some macroscopic models accounting for diffusion (without claiming a thorough analysis of every such model described in the available literature). 
(a) Models accounting for the surface diffusion. One such model was considered in the description of a topochemical model [112]. A correct mathematical description of multicomponent diffusion processes in solids obtained on the basis of irreversible process thermodynamics can be found in refs. 125127.

This description shows first, that due to the independent character of elementary diffusion acts between atoms of various substances, their diffusion mobility is controlled by the different partial diffusion coefficients; second, that the diffusion of atoms and molecules adsorbed on the surface takes place due to their "overjumps" to neighbouring unoccupied sites (vacancies).

Thus, for the oxidation of $\mathrm{CO}$ on a metal wire the model accounting for the surface diffusion is of the form [128]

$$
\begin{aligned}
& \dot{x}=W_{\text {ads }, \mathrm{O}_{2}}-W_{\text {des }, \mathrm{O}_{2}}-W_{\text {react }} \\
& \dot{y}=W_{\text {ads }, \mathrm{CO}}-W_{\text {des, } \mathrm{CO}}-W_{\text {react }}+D(z \Delta y-y \Delta z)
\end{aligned}
$$

where $x, y, z$ are the concentrations of $\mathrm{MeO}, \mathrm{MeCO}$ and $\mathrm{Me}(z=1-x-y)$, respectively, $W_{\mathrm{ads}, \mathrm{O}_{2}}$ and $W_{\mathrm{ads}, \mathrm{CO}}$ the adsorption rates on catalysts of $\mathrm{O}_{2}$ and $\mathrm{CO}$, respectively, $W_{\mathrm{des}, \mathrm{O}_{2}}$ and $W_{\mathrm{des}, \mathrm{CO}}$ the desorption rates, $W_{\text {react }}$ the reaction rate between the adsorbed species, $D$ the diffusion coefficient, $\Delta$ the Laplacian operator, in the one-dimensional case

$$
\Delta x=\frac{\partial^{2} x}{\partial \xi^{2}} ; \quad \Delta y=\frac{\partial^{2} y}{\partial \xi^{2}} ; \quad \Delta z=\frac{\partial^{2} z}{\partial \xi^{2}}
$$

and $\xi$ the wire coordinate.

This model will primarily account for the resolving of local activity "spots" during the reaction. But in models of such kind, periodic spatial structures ("dissipative structures") can also be formed and these have recently become of great interest.

The effect of surface diffusion on the selectivity of the catalytic reaction $\mathrm{A} \rightarrow \mathrm{B} \rightarrow \mathrm{C}$ has been examined [129]. The authors suggest that the sites of $\alpha$-phase (assumed to be round) are uniformly distributed over the $\beta$-phase. Reaction A $\rightarrow$ B takes place only on the $\alpha$-phase, whereas B $\rightarrow$ C occurs only on the $\beta$-phase. Substance B formed on the $\alpha$-phase is transferred to the $\beta$-phase due either to surface diffusion or adsorption-desorption processes.

These conceptions are met by a sufficiently simple model which is a set of three differential equations (two balance equations for substances $\mathrm{A}$ and $\mathrm{B}$ on the $\alpha$-phase and one equation for substance B on the $\beta$-phase).

It was shown that surface diffusion can exert a strong influence on reaction selectivity. Of interest is the result obtained in studying the model [129], i.e. the dependence of selectivity on the crystallite sizes of the $\alpha$-phase.

In several studies by Kaminskii and his co-workers [130-133], the time dependences of heterogeneous processes involving reactants migrating over the surface was described by the diffusion kinetics equations but the par- 
ameters to determine the probability of elementary chemical acts enter into the corresponding boundary conditions.

Thus Kaminskii et al. [133] considered the kinetics of monomolecular surface reactions taking place on the boundary of immovable active centres (circles having a radius $r_{\mathrm{o}}$ ) which form a square lattice with the constant, $L$. The diffusion coefficient, $D$, is represented as $D=p a^{2}$ where $p$ is the probability of a jump per unit time and $a$ the respective parameter of the lattice size.

A criterion was obtained [133] under the fulfilment of which the diffusion can be treated as rapid and not taken into consideration for the surface reaction kinetics: $k /(a p)\left[\ln \left(L / r_{\mathrm{o}}\right)-1.39\right] \ll 1$, where $k$ is the interaction constant of adsorbed substances with active centres. It is evident that at $L \approx r_{0}$ this relationship is met. It is this relationship that is the condition for the applicability of the ideal adsorbed layer kinetics but all the limitations imposed for its derivation (the reaction is monomolecular and active centres are taken for a square lattice) should be remembered.

(b) Models accounting for the surface-to-bulk mass transfer. At present a great number of experimental data have been accumulated that give evidence to the effect of mass transfer into the solid catalyst body on the character of catalytic reaction processes [134-136].

The simplest model of such a process acounting for the surface reactions and diffusion of a single substance is [137-139]

$$
\begin{aligned}
& \frac{\partial c}{\partial t}=\frac{D}{L^{2}} \frac{\partial^{2} c}{\partial \xi^{2}} \quad 0<\xi<1 \\
& \xi=0: \quad \frac{\partial c}{\partial \xi}=0 ; \\
& \xi=1: \quad c=\frac{C_{z}}{C_{\mathrm{v}}} H x_{1} \\
& \frac{\mathrm{d} x_{1}}{\mathrm{~d} t}=\mathrm{f}_{1}\left(x_{1}, \ldots, x_{m}\right)-\frac{D C_{\mathrm{v}}}{L C_{\mathrm{z}}}\left[\frac{\mathrm{d} c}{\mathrm{~d} \xi}\right]_{\xi=1} \\
& \frac{\mathrm{d} x_{i}}{\mathrm{~d} t}=\mathrm{f}_{i}\left(x_{1}, \ldots, x_{m}\right) \quad i=2, \ldots, m \\
& t=0: \quad x_{i}=x_{i}^{\circ} \quad i=1, \ldots, m \\
& c=c_{0}(\xi)
\end{aligned}
$$

where $\xi$ is a dimensionless coordinate, $x_{i}$ the dimensionless concentrations of substances on the catalyst surface, $c$ the dimensionless concentration of the substance diffusing into catalyst bulk, $D$ the diffusion coefficient of this substance $\left(\mathrm{cm}^{2} \mathrm{~s}^{-1}\right), L$ the thickness of the diffusion layer $(\mathrm{cm}), C_{z}$ the number of active centres on unit catalyst surface (molecules $\mathrm{cm}^{-2}$ ), $C_{\mathrm{v}}$ the maximum possible number of diffusing reactant particles per unit catalyst volume (molecules $\mathrm{cm}^{-3}$ ), and $H$ a parameter associated with the crystal lattice geometry $\left(\mathrm{cm}^{-1}\right)$. 
Strictly speaking, in the case in which more than one substance diffuses, the equation

$$
\frac{\partial c}{\partial t}=\frac{D}{L^{2}} \frac{\partial^{2} c}{\partial \xi^{2}}
$$

cannot be used. A correct description of the multicomponent diffusion in solids should be used [125, 126]. Merzhanov and Bloshenko [140] represented mass transfer as a reversible surface-to-bulk exchange process. Such models can be modified by representing diffusion as a reversible substance exchange process between a sequence of layers, the first being the catalyst surface. In these models it is not necessary to use the simplified relationship $c=C_{2} H x_{1} /$ $C_{\mathrm{v}}$ which permits us to go from bulk to surface concentrations that are found from solving the problem.

A detailed study of model (16) for $\mathrm{CO}$ oxidation on polycrystalline platinum was carried out by Makhotkin et al. [139]. Numerical experiments revealed that the bulk diffusion effect on the character of reaction dynamics is rather different and controlled by the following factors: (1) the initial composition of catalyst surface and bulk, (2) the steady state of its surface and bulk, and (3) the position of the region for slow relaxations of kinetic origin (see ref. 139). As a rule, diffusion retards the establishment of steady states, but the case in which the attainment of this state is accelerated by diffusion is possible.

Thus, from the consideration of some models accounting for the diffusion either on the catalyst surface or in its bulk, one can draw the conclusion that no systematic experience in the application of such models has been accumulated.

\subsection{HETEROGENEOUS-HOMOGENEOUS CATALYTIC REACTION MODELS}

Some concepts of the homogeneous-heterogeneous process of complex reactions have been obtained from a series of investigations by Polyakov and his school [141]. Some results were reported in refs. 142 and 143.

On the other hand, from studies by Azatyan (see, for example, refs. 144146) it becomes clear that, in the course of branching chain processes, the solid phase in contact with the reaction mixture changes significantly. Essential changes are also observed in the mechanisms and kinetics for the heterogeneous decay of active centres. Previously it was believed that the "rate constant" of heterogeneous chain termination on walls remains unchanged during the oxidation process, but now heterogeneous chain termination should be regarded as a complex reaction catalyzed by the wall.

Under certain conditions, the catalyst surface can be a source for the formation of intermediates evolving into the gas phase. These intermediates can initiate chain reactions.

Thus the logic of studies forces us to take into account the formation of homogeneous constituents in heterogeneous catalytic reactions and heterogeneous constituents in homogeneous processes. 
As far as the models accounting for these conceptions are concerned, their construction and investigation have just started. The development of these models is sure to be retarded by the absence of data on the detailed reaction mechanism and its parameters. The exception is ref. 147, where the authors construct an unsteady-state homogeneous-heterogeneous reaction model and analyze it with respect to the cyclohexane oxidation on zeolites. The study was aimed at the experimental interpretation of the self-oscillations found. The model constructed is in accordance with the law of mass action.

\subsection{PHENOMENOLOGICAL MODEL OF BRANCHED-CHAIN REACTIONS ON A CATALYST SURFACE}

In the 1950s, Semenov and Voevodskii [148] made an attempt to apply the concepts of the branching-chain reaction theory to the kinetics of heterogeneous catalysts. They applied the concept of free valencies migrating over the catalyst surface and of "semi-chemisorbed" radicals. But their attempt was criticized (see, for example, ref. 149 where Temkin, using hydrogenation of ethylene on palladium as an example, proved experimentally the inapplicability of the chain theory concepts).

Recently, Barelko et al. [150-155] put forward a new version of this theory. They suggested a branching-chain process mechanism based on the concepts implying the existence of a two-dimensional gas of adsorbed atoms (adatoms) on solid surfaces which are in equilibrium with their crystal lattice. According to the suggested hypothesis, the active centre is the adatom. The energy evolved in the course of a reaction on the adatom can be applied to break out another atom from the lattice, i.e. to form a new active centre. This process is a step of branching. But the decay of the active centre takes place due to the return of the adatom back into the lattice.

At present the literature lacks strong experimental proof for this hypothesis which was put forward to interpret the critical effects seen in catalytic oxidation reactions. As far as the model itself is concerned, it is of the form of ref. 150 , which is similar to the chain reaction equation

$$
\frac{\mathrm{d} n}{\mathrm{~d} t}=W_{\mathrm{o}}+\mathrm{F}(n)-\left.\mathrm{G}(n) \quad n\right|_{t=0}=n_{\mathrm{o}}
$$

where $n$ and $n_{\mathrm{o}}$ are the current and initial concentrations of the active centres, $W_{\mathrm{o}}$ is the initiation rate, and $\mathrm{F}(n)$ and $G(n)$ are the rates of active centre generation and decay.

\section{Conclusion}

In conclusion let us present some simple considerations that are, to our mind, of importance. Heterogeneous catalytic reaction is a complex process 
determined by the interaction of various factors. Depending on the reaction conditions, one must apply different models of various complexity. This is quite natural: similar ideology is systematically followed, for example, in modelling catalytic processes and reactors [156].

We believe that a developed system of models to describe the steps of complex catalytic reactions has not yet been constructed. A combination of some particular models described in this section is quite naturally of a non-systematic and incomplete character.

It should be noted that the detailed modelling of heterogeneous catalytic reactions faces some specific difficulties. Compared with homogeneous systems, the limits of the field wherein the law of mass action analog (the surface-action law) can be correctly applied are less distinct. Still less reliable are the elementary step constants. Nevertheless, we believe that, despite the complexity of "real kinetics", the importance of studying the models fitting the law of mass action cannot be undervalued. These models describe the chemical components of a complex catalytic process properly and, on the other hand, they are a necessary step that can be treated as a first approximation. Our study is devoted to the analysis of just these models.

\section{References}

1 V.A. Kritsman, Development of Organic Reaction Kinetics, Nauka, Moscow, 1970 (in Russian).

2 W. Ostwald, Evolution of the Principal Problems of Chemistry, Moscow, 1906 (in Russian).

3 A. Mittasch and E. Theiss, Von Davy und Döbereiner bis Deakon, ein halbes Jahrhundert Grenzflächenkatalyse, Leipzig, 1915 (ONTI, Khar'kov, 1934 (in Russian)).

4 J.H. Van't Hoff, Etudes de Dynamique Chimique, Frederic Muller, Amsterdam, 1884 (ONTI - Khim Teoret, Leningrad, 1936 (in Russian)).

5 N.N. Semenov, Evolution of the Theory of Chain Reactions and Thermal Ignition, Znanie, Moscow, 1969 (in Russian).

6 L.S. Polak, Non-equilibrium Chemical Kinetics and its Application, Nauka, Moscow, 1979 (in Russian).

7 V.I. Kouzmin, B.D. Lebedev and Yu.V. Chuev, Probl. Kibern., 24 (1971) 5.

8 Yu.M. Svirezhev and D.O. Logofet, Stability of Biological Populations, Nauka, Moscow, 1978 (in Russian).

9 N.S. Pechurkin, Population Microbiology, Nauka, Novosibirsk, 1978 (in Russian).

10 A.J. Lotka, Elements of Physical Biology, Williams and Wilkins, Baltimore, 1925.

11 A.J. Lotka, Elements of Mathematical Biology, Dover, New York, 1956.

12 V. Volterra, Leçóns sur la Théorie Mathématique de la Lutte pour la Vie, GauthiersVillars, Paris, 1931.

13 J. Horiuti, Physical Chemistry Problems, Goskhimizdat, Moscow, 1953, pp. 39-55 (in Russian).

14 E. Christiansen, Catalysis. Investigation of Heterogeneous Processes, Inostrannaya Literatura, Moscow, 1956, pp. 135-237 (in Russian).

15 Ya.G. Dorfman, Lavoisier, Izd. Akad. Nauk SSSR, Moscow, 1962 (in Russian).

16 V.A. Kritsman, Structural Kinetic Regularities, Nauka, Moscow, 1974 (in Russian). 
17 A.Ya. Kipnis, Guldberg and his Contribution to the Development of Physical Chemistry, Izd. Akad. Nauk SSSR, Moscow, 1963, pp. 329-369 (in Russian).

18 J.K. Maxwell, Articles and Reports, Nauka, Moscow, 1968 (in Russian).

19 G.B. Dobrotin and Yu. I. Soloviev, Van't Hoff, Nauka, Moscow, 1977 (in Russian).

20 M.G. Slin'ko and G.S. Yablonskii, Probl. Kinet. Katal., 17 (1978) 154.

21 E.E. Nikitin, Kinetic Problems of Elementary Chemical Reactions, Nauka, Moscow, 1973 , pp. 51-60 (in Russian).

22 S. Glasstone, K.J. Laidler and H. Eyring, The Theory of Rate Processes, Princeton University Press, New York and London, 1941.

23 Proceedings of the Symposium on Reaction Mechanisms. Models and Computers, J. Phys. Chem., 81 (1977) 2309-2586.

24 Proceedings of the Symposium on Current Status of Kinetics of Elementary Gas Reactions. Predictive Power of Theory and Accuracy of Measurements, J. Phys. Chem., 83 (1979) 1-207.

25 I.I. Blekhman, A.D. Myshkis and Ya.G. Panovko, Applied Mathematics, Subject, Logics, Specificity of Approaches, Naukova Dumka, Kiev, 1976 (in Russian).

26 G.S. Yablonskii and S.I. Spivak, Mathematical Models of Chemical Kinetics, Znanie, Moscow, 1977 (in Russian).

27 G.K. Boreskov and M.G. Slin'ko, Vestn. Akad. Nauk SSSR, 10 (1961) 29.

28 G.K. Boreskov and M.G. Slin'ko, Teor. Osn. Khim. Tekhnol., 1 (1967) 5.

29 V.I. Dimitrov, React. Kinet. Catal. Lett., 7 (1977) 81.

30 V.I. Dimitrov, Simple Kinetics, Nauka, Novosibirsk, 1982 (in Russian).

31 L.S. Polak, M.Ya. Goldenberg and A.A. Levitskii, Computational Methods in Chemical Kinetics, Nauka, Moscow, 1984 (in Russian).

32 N.N. Semenov, Chain Reactions, Goskhimizdat, Leningrad, 1934 (in Russian).

33 V.A. Engelhardt, Vopr. Philos., 7 (1976) 65.

34 I. Langmuir, J. Am. Chem. Soc., 37 (1915) 1139; 38 (1916) 2221.

35 I. Langmuir, Trans. Faraday Soc., 17 (1922) 607, 621.

36 M.I. Temkin, Zh. Fiz. Khim., 11 (1938) 169.

37 Yu.S. Snagovskii and G.M. Ostrovskii, Kinetic Modelling of Heterogeneous Catalytic Processes, Khimiya, Moscow, 1976 (in Russian).

38 O.V. Krylov, Kinetic Problems of Elementary Chemical Reactions, Nauka, Moscow, 1973, pp. 115-130 (in Russian).

39 G.I. Golodets and V.A. Roiter, Kinet. Katal., 13 (1972) 590.

40 R.W. Maatman, Catal, Rev., 8 (1973) 1.

41 R.W. Maatman, J. Catal., 43 (1976) 1.

42 S.L. Kiperman, Fundamentals of Chemical Kinetics in Heterogeneous Catalysis, Khimiya, Moscow, 1979 (in Russian).

43 J. Horiuti, J. Res. Inst. Catal. Hokkaido Univ., 5 (1957) 1.

44 J. Horiuti and T. Nakamura, Z. Phys. Chem. N.F., 11 (1957) 358.

45 M.I. Temkin, Dokl. Akad. Nauk SSSR, 152 (1963) 156.

46 M.I. Temkin, Mechanism and Kinetics of Composite Catalytic Reactions, Nauka, Mos* cow, 1970, pp. 57-72 (in Russian).

47 M.I. Temkin, Zh. Vses. Khim. Ova., 20 (1975) 7.

48 V.N. Kondratiev and E.E. Nikitin, Kinetics and Mechanism of Gas-Phase Reactions, Nauka, Moscow, 1974 (in Russian).

49 A.R. Hochstim (Ed.), Kinetic Processes in Gases and Plasma, Academic Press, New York, London, 1969.

50 E.E. Nikitin, Theory of Elementary Atomic-Molecular Processes in Gases, Khimiya, Moscow, 1970.

51 L.S. Polak, Non-equilibrium Chemical Kinetics and Its Applications, Nauka, Moscow, 1979 (in Russian).

52 V.P. Zhdanov, Dokl. Akad. Nauk SSSR, 253 (1980) 522. 
53 V.P. Zhdanov and K.I. Zamaraev, Catal. Rev. Sci. Eng., 24 (1982) 373.

54 H.S. Taylor, Proc. R. Soc. London Ser. A, 108 (1925) 105; J. Phys. Chem., 30 (1925) 145.

55 M.I. Temkin, Zh. Fiz. Khim., 15 (1941) 296.

56 M.I. Temkin, Zh. Fiz. Khim., 14 (1940) 1153.

57 S.Z. Roginskii and Ya.B. Zeldovich, Acta Phys. Chim. USSR, 1 (1934) 554.

58 Ya.B. Zeldovich, Acta Phys. Chim. USSR, 1 (1935) 961.

59 M.I. Temkin and V.M. Pyzhov, Zh. Fiz. Khim., 13 (1939) 857.

60 S.Z. Roginskii, Adsorption and Catalysis on Inhomogeneous Surfaces, Izd. Akad. Nauk SSSR, Moscow, Leningrad, 1948 (in Russian).

61 S.L. Kiperman, Introduction in the Kinetics of Heterogeneous Catalytic Reactions, Nauka, Moscow, 1964 (in Russian). .

62 M. Boudart, J. Am. Chem. Soc., 74 (1952) 3556.

63 I. Langmuir, J. Am. Chem. Soc., 54 (1932) 2798.

64 J.H. de Boer, Advances in Catalysis and Related Subjects, Academic Press, New York, 1956.

65 M.I. Temkin, Kinet. Katal., 13 (1972) 555.

66 T.S. Jayadevaiah and R.Vancelow (Eds), Surface Science: Recent Progress and Perspectives, CRC Press, Cleveland, 1974 [Mir, Moscow, 1977 (Russian edition)].

67 A.W. Czanderna (Ed.), Methods of Surface Analysis, Vol. I, Elsevier, Amsterdam, 1975.

68 G.K. Boreskov, Kinet. Katal., 13 (1972) 543.

69 M. Boudart, A.W. Aldag, L.D. Ptak and J.E. Benson, J. Catal., 11 (1968) 35.

70 G.K. Boreskov, Theoretical Problems of Catalysis, Nauka, Novosibirsk, 1977, pp. 113-120 (in Russian).

71 V.N. Shtyrkov, G.A. Kozlova, R.P. Kayumov, Yu.E. Roginskaya, N.V. Kulkova, A.S. Sadovskii and A.I. Gel'bshtein, in Proc. 2nd All-Union Conf. Kinet. Catal. React. (Kinetics-2), Vol. 1, Institute of Catalysis, Novosibirsk, 1975, pp. 119-126 (in Russian).

72 R.I. Maksimovskaya, S.A. Venyaminov and G.K. Boreskov, Dokl. Acad. Nauk SSSR, 211 (1973) 1389.

73 L.P. Levchenko, N.V. Kulkova and M.I. Temkin, Kinet. Katal., 17 (1976) 1542.

74 V.A. Sadykov and P.G. Tsyrulnikov, Kinet. Katal., 17 (1976) 618.

75 S.A. Venyaminov, A.N. Pitaeva, G.B. Barannik, L.M. Plyasova, R.I. Maksimovskaya and G.N. Kustova, Kinet. Katal., 18 (1977) 456.

76 M. Flytzani-Stephanopoulos and L.D. Schmidt, Prog. Surf. Sci., 9 (1979) 83.

77 V.I. Bykov, A.V. Fedotov, Yu.Sh. Matros, G.S. Yablonskii, Yu.I. Kuznetsov and M.G. Slin'ko, Teoret. Osn. Khim. Tekhnol., 8 (1974) 378.

78 Ch. V. Kopetskii, Vestn. Akad. Nauk SSSR, 9 (1979) 10.

79 H.P. Bonzel, Surf. Sci., 68 (1977) 236.

80 G.A. Somorjai, Annu. Rev. Phys. Chem., 19 (1968) 251.

81 L.D. Schmidt, in R. Gomer (Ed.), Interactions on Metal Surfaces, Springer Verlag, Berlin, Heidelberg, 1975.

82 L.A. Bolshov, A.P. Napartovich, A.G. Naumovets and A.G. Fedorus, Usp. Fiz. Nauk, 122 (1977) 125.

83 V.I. Savchenko, Kinet. Katal., 21 (1980) 832.

84 G.K. Boreskov and V.I. Savchenko, Commun. Dep. Chem., Bulg. Acad. Sci,, 16 (1983) 57.

85 D.G. Castner and G.A. Somorjai, Chem. Rev., 79 (1979) 233.

86 C.G. Goymour and D.A. King, J. Chem. Soc. Faraday Trans. 1, 69 (1973) 736, 749.

87 D.L. Adams, Surf. Sci., 42 (1974) 12.

88 Yu.K. Tovbin and V.K. Fedyanin, Kinet. Katal., 19 (1978) 989, 1202.

89 Yu.K. Tovbin, Kinet. Katal., 19 (1978) 1453; 20 (1979) 1226.

90 P.A. Zhdan, G.K. Boreskov, W.F. Egelhoff, Jr. and W.H. Weinberg, Surf. Sci, 61 (1976) 377.

91 T. Engel and G. ErtI, J. Chem. Phys., 69 (1978) 1267.

92 J.L. Taylor, D.E. Ibbotson and W.H. Weinberg, Surf. Sci., 90 (1979) 37.

93 Yu.K. Tovbin, Dokl. Akad. Nauk SSSR, 260 (1981) 679. 
94 M.G.De Oliveira and R.B. Griffiths, Surf. Sci., 7 (1978) 687.

95 M. Wilf and P.T. Dawson, Surf. Sci., 65 (1977) 399.

96 G. Doyen and G. Ertl, J. Chem. Phys., 62 (1975) 2953.

97 L.D. Roelofs, R.L. Park and T.L. Einstein, J. Vac. Sci. Technol., 16 (1979) 478.

98 M. Bowker and D.A. King, Surf. Sci., 71 (1978) 583.

99 H.P. Bonzel and R. Ku, Surf. Sci., 40 (1973) 85.

100 C. Wang and R. Gomer, Surf. Sci., 84 (1979) 329.

101 E.W. Montroll, Stability and Phase Transitions, Mir, Moscow 1973 (in Russian).

102 T.L. Einstein, Solid State Mater. Sci., 7 (1978) 261.

103. V.P. Zhdanov, Surf. Sci., 102 (1981) L35.

104 V.P. Zhdanov, Surf. Sci., 111 (1981) 63.

105 R.D. Young and D.C. Schubert, J. Chem. Phys., 42 (1965) 3943.

106 S.E. Gilev, A.N. Gorban, V.I. Bykov and G.S. Yablonskii, Dokl. Akad. Nauk SSSR, 262 (1982) 1413.

107 G. Ertl and D. Schillinger, J. Chem. Phys., 66 (1977) 2563.

108 K. Binder and D.P. Landau, Surf. Sci., 61 (1976) 577.

109 E.D. Williams, S.L. Cunningham and W.H. Weinberg, J. Chem. Phys., 60 (1978) 4688.

110 D.M. Lu, Surf. Sci., 93 (1980) L111.

111 B. Delmon, Introduction à la Cinétique Hétérogène, Technip, Paris, 1969.

112 P.H. Holloway and J.B. Hudson, Surf. Sci., 43 (1974) 123.

113 A.Ya. Rozovskii, Kinetics of Topochemical Reactions, Khimiya, Moscow, 1974 (in Russian).

114 A.D. Berman and O.V. Krylov, Probl. Kinet. Katal., 17 (1978) 102.

115 B.V. Erofeev, Dokl. Akad. Nauk SSSR, 52 (1946) 515.

116 G.K. Boreskov, V.I. Savchenko, K.A. Dadayan, V.P. Ivanov and N.N. Bulgakov, Probl. Kinet. Katal., 17 (1978) 115.

117 V.I. Savchenko, V.P. Ivanov and K.A. Dadayan, Proc. 5th Soviet-Japanese Seminar on Catalysis, FAN UzSSR, Tashkent, 1979, pp. 244-251 (in Russian).

118 V.I. Savchenko, Mechanism of Catalysis. Part 2: Investigation Methods for Catalytic Reactions, Nauka, Novosibirsk, 1984, pp. 126-142 (in Russian).

119 A.M. Kolchin, Z.F. Kolchina and V.F. Malakhov, Proc. 2nd All-Union Conf. Kinet. Catal. React. (Kinetics-2), Vol. 3, Institute of Catalysis, Novosibirsk, 1975, pp. 51-57 (in Russian).

120 K.A. Dadayan, G.K. Boreskov, V.I. Savchenko, E.M. Sadovskaya and G.S. Yablonskii, Kinet. Katal., 20 (1979) 795.

121 W.J. Dunning, Nucleation, Dekker, New York, 1969.

122 D. Robertson and G.M. Pound, in I.S. Jayadevaiach and R. Vancelow (Eds.), Surface Science: Recent Progress and Perspectives, Part 1, CRC Press, Cleveland, 1974 [Mir, Moscow, 1977, pp. 64-151 (Russian edition)].

123 C.N. Sutterfield and T.K. Sherwood, The Role of Diffusion in Catalysis, Addison-Wesley, Reading, MA, 1965.

124 R. Aris, The Mathematical Theory of Diffusion and Reaction in Permeable Catalysts, Clarendon Press, Oxford, 1975.

125 I.B. Borowskii, K.P. Gurov and I.D. Marchukova, Mutual Diffusion Processes in Alloys, Nauka, Moscow, 1973 (in Russian).

126 Ya.E. Geguzin, Diffusional Zone, Nauka, Moscow, 1979 (in Russian).

127 V.Ya. Mariengof and Yu.A. Taranenko, Chemistry of Oxide Catalyst Surfaces, Nauka, Moscow, 1979, pp. 83-95 (in Russian).

128 A.N. Gorban, V.I. Bykov and G.S. Yablonskii, Chem. Eng. Sci., 35 (1980) 2351.

129 H.H. Kung and M.C. Kung, Chem. Eng. Sci., 33 (1978) 1003.

130 P.A. Vrunov, V.A. Kaminskii and S.F. Timashev, Teor. Eksp. Khim., 10 (1974) 380.

131 A.A. Ovchinnikov and S.F. Timashev, Dokl. Akad. Nauk SSSR, 239 (1980) 643.

132 P.A. Vrunov, V.A. Kaminskii and S.F. Timashev, Kinet. Katal., 19 (1978) 1295. 
133 V.A. Kaminskii, B.N. Okunev and A.A. Ovchinnikov, Dokl. Akad. Nauk SSSR, 251 (1980) 636.

134 M.I. Temkin, Kinet. Katal., 17 (1976) 1095.

135 B.S. Balzhinimaev, M.A. Shmeleva, V.I. Timoshenko and G.R. Kotelnikov, Proc. 2nd All-Union Conf. Kinet. Catal. React. (Kinetics-2), Vol. 3, Institute of Catalysis, Novosibirsk, 1975, pp. 85-91 (in Russian).

136 C.T. Campbell, D.C. Foyt and J.M. White, J. Phys. Chem., 81 (1977) 491.

137 D.E. Beck and E. Miyazaki, Surf. Sci., 39 (1973) 37; 48 (1975) 473.

138 H.C. Chang and W.H. Weinberg, Surf. Sci., 65 (1977) 153.

139 O.A. Makhotkin, V.I. Elokhin and G.S. Yablonskii, Proc. 3rd All-Union Conf. Math. Methods Chem., Vol. 1, TsNIITEneftekhim, Moscow, 1980, pp. 77-85 (in Russian).

140 A.G. Merzhanov and V.I. Bloshenko, Dokl. Akad, Nauk SSSR, 242 (1978) 1118.

141 M.V. Polyakov, Heterogeneous Catalysis in the Chemical Industry, Gos. NauchnoTekhn. Izd. Khim. Lit., Moscow, 1955, pp. 271-280 (in Russian).

142 Ya.B. Gorokhovatskii, T.P. Kornienko and V.V. Shalya, Heterogeneous-Homogeneous Reactions, Tekhnika, Kiev, 1972 (in Russian).

143 L.Ya, Margolis, Hydrocarbon Oxidation by Heterogeneous Catalysts, Khimiya, Moscow, 1977.

144 V.V. Azatyan and N.N. Semenov, Problems of Chemical Kinetics, Nauka, Moscow, 1980, pp. 5-21 (in Russian).

145 V.V. Azatyan, Fiz. Goreniya Vzryva, 11 (1975) 256.

146 V.V. Azatyan, Kinet. Katal., 18 (1977) 1098.

147 A.D. Berman and A.V. Elinek, Dokl. Akad. Nauk SSSR, 248 (1979) 643.

148 N.N. Semenov and V.V. Voevodskii, Heterogeneous Catalysis in the Chemical Industry, Gos. Nauchno-Tekhn. Izd. Khim. Lit., Moscow, 1955, pp. 233-256 (in Russian).

149 M.I. Temkin, Commun. Dep. Chem., Bulg. Acad. Sci., 1 (1968) 65.

150 V.V. Barelko and Yu.E. Volodin, Dokl. Akad. Nauk SSSR, 216 (1974) 1080.

151 V.V. Barelko and Yu.E. Volodin, Kinet. Katal,, 17 (1976) 683.

152 V.V. Barelko and Yu.E. Volodin, Dokl. Akad. Nauk SSSR, 223 (1975) 362.

153 Yu.E. Volodin, V.V. Barelko and P.I. Khalzov, Dokl. Akad. Nauk SSSR, 234 (1977) 124.

154 V.V. Barelko and A.G. Merzhanov, Probl. Kinet. Katal., 17 (1978) 182.

155 V.V. Barelko, Probl. Kinet. Katal., 18 (1981) 61.

156 M.G. Slin'ko, Modelling of Chemical Reactors, Nauka, Novosibirsk, 1968 (in Russian). 